\title{
A generic hybrid model for bulk elastodynamics, with application to ultrasonic Non-Destructive Evaluation
}

\author{
P. Rajagopal, E. A. Skelton, W. Choi, M. J. S. Lowe and R. V. Craster
}

\begin{abstract}
Practical ultrasonic inspection requires modeling tools that enable rapid and accurate visualisation; due to the increasing sophistication of practical inspection it is becoming increasingly difficult to use a single modelling method to represent an entire inspection process. Hybrid models that utilize different or interacting numerical schemes in different regions, to use their relative advantages to maximal effect, are attractive in this context, but are usually custommade for bespoke applications or sets of modelling methods. The limitation of hybrid schemes to particular modelling techniques is shown here to be related to their fundamental formulation. As a result it becomes clear that a formalism to generalize hybrid schemes can be developed: an example of how such a generic hybrid modelling interface is constructed is illustrated for the abstraction of bulk ultrasonic wave phenomena, common in practical inspection problems. This interface is then adapted to work within a prototype hybrid model consisting of two smaller Finite Element model-domains, and explicitly demonstrated for bulk ultrasonic wave propagation and scattering examples. Sources of error and ways of improving the accuracy of the interface are also discussed.
\end{abstract}

\section{INTRODUCTION}

Simulators capable of modelling an entire inspection process are of abiding interest to the ultrasonic Non Destructive Evaluation (NDE) community; the creation of fast, accurate and efficient numerical techniques is becoming even more important given the ever increasing sophistication of practical ultrasonic inspection. A desirable goal is to model the complete experimental situation leading to the direct comparison of simulation with measurement. Such tools are also attractive for inspection qualification in industries with stringent safety standards such as nuclear power generation. The ever increasing complexity and scale of the features to be inspected, and consequently of the transduction, make it increasingly challenging to apply a single modelling method to an entire inspection process. The aim of this article is to present a generic methodology capable of addressing these issues.

\footnotetext{
P. Rajagopal, W. Choi and M. J. S. Lowe are with the Department of Mechanical Engineering, Imperial College London

P.Rajagopal current address Mechanical Engineering Department, Indian Institute of Technology - Madras, Chennai 600036, T.N., India

E. A. Skelton and R. V. Craster are with the Department of Mathematics, Imperial College London, London U.K.

Manuscript received March 1, 2012
} 
Over many years mesh-based numerical solution procedures such as Finite Element (FE) and Finite Difference (FD) methods have emerged as important tools in the quantitative analysis of ultrasonic wave problems, especially in studying scattering phenomena [1]-[4]. Robust and efficient commercial packages are now widely available, (for example, [5] ABAQUS, [6] PZFlex and [7] Comsol) and provide good support as well as allowing for the rapid transfer of techniques to industry. Solutions to some key past impediments to the numerical modelling of wave phenomena, such as representing unbounded domains or complex features and geometries have now become accessible to the wider community via Perfectly Matched Layers and absorbing layers [4], [8]-[11] and have been directly implemented in commercial packages [12], [13]. However, these methods still lead to an enormous computational cost when dealing with large volumes of material and representing complex materials; modelling realistic transduction also remains a challenge. Moreover each defect study requires a specific model and thus many numerically intensive runs are required to understand scattering from multiple defects or defect configurations, even if the transduction remains the same. Commercial alternatives to such purely numerical schemes, (for example, analytical solution and ray-tracing based software such as CIVA, [14]) do exist and are able to handle a range of transduction methods and wave propagation through complex features; however modelling wave scattering from complex defects remains a challenge for these methods.

To handle this increasing complexity of inspection one can treat the total process as a series of modules comprising wave excitation, scattering, reception, and post-processing, as for example, shown in Figure 1. Then the relative merits of different methods in dealing with one or more of such modules are harnessed by linking them up in a global 'hybrid' model; a long history within the ultrasonic NDE community [15]-[18] of such models exists. But this effort was primarily devoted to specific applications or problems, or particular combinations of analytical or numerical methods, and thus required the development and maintenance of specialist codes to implement them. Similarly the medical ultrasound community has approached FE-FE meshes in the past [19], however that was limited to simpler acoustic/acoustic coupling only and not the more detailed solid/solid hybrid methods that are required for coupled NDE simulations.

The aim of the current article is to develop general procedures that allow the creation of hybrid models combining any set of chosen modelling methods. It is also the aim to arrive at methods that can be readily implemented without modifying the underlying modelling procedures, therefore allowing commercial packages to be utilized. As a first step, in this paper we present the development and validation of such an approach for two-dimensional cases.

This paper is organized as follows. Section II provides a description of the key step in achieving hybrid models, that is, the decomposition of the total inspection process into a number of constituent modules. In view of our interest in generalizing this process, the basis and validity of this modular approach is discussed, incorporating insights from other disciplines where a similar process is employed. In Section III an integral-representation based formalism for hybrid models is developed, showing how they become limited to specific sets of underlying methods. A generalization is shown to emerge from conditions where modules comprising wave excitation, scattering and reception can further be split and inter-related by an intermediate, wave propagation module. Section IV develops a scheme for such a generic wave propagator module for ultrasonic wave phenomena in the bulk of an infinite 
homogeneous isotropic medium; this uses the fundamental physics of the problem permitting analysis in two dimensions. An important aim of this article is to have a formulation that is implementable using standard commercial software; this, and validation of the scheme is illustrated in Section V. A prototype hybrid model is considered, where, for simplicity, the FE method is used to represent domains enclosing both the wave excitation and the forward locations. This adaptation is validated against results from full-FE simulations of wave propagation and scattering by a simple barrier, and then a realistic practical example is shown, comprising scattering from a row of side-drilled holes. Finally, the paper ends with discussion and concluding remarks in Section VI.

\section{BACKGROUND}

Hybrid models have a long history within the context of elastic wave scattering studies necessitated by the intrinsic underlying difficulty of having two, often disparate, characteristic dimensions occurring simultaneously, namely the wavelength and the characteristic defect dimension [20]. Elastic wave scattering from a defect has to satisfy Navier's equation of motion,

$$
\frac{\partial \sigma_{i j}}{\partial x_{j}}+f_{i}-\rho \frac{\partial^{2} u_{i}}{\partial t^{2}}=0
$$

or equivalently,

$$
C_{i j k l} \frac{\partial^{2} u_{k}}{\partial x_{j} \partial x_{l}}-\rho \frac{\partial^{2} u_{i}}{\partial t^{2}}+f_{i}=0
$$

as well as the conditions on the boundaries of artifacts or obstacles, which in NDE applications are usually either those of a

$$
\begin{gathered}
\text { cavity (zero traction) } \rightarrow\left(\sigma_{i j} n_{j}\right)^{\text {boundary }}=0 \text { or } \\
\text { inclusion (continuous fields) } \rightarrow\left(u_{i}\right)^{\text {substrate }}=\left(u_{i}\right)^{\text {inclusion }}
\end{gathered}
$$

and

$$
\left(\sigma_{i j} n_{j}\right)^{\text {substrate }}=\left(\sigma_{i j} n_{j}\right)^{\text {inclusion }}
$$

where $\sigma_{i j}$ denotes the ij component of the stress tensor, $n_{i}$ is the $\mathrm{i}$ th component of the outward pointing normal to the defect, $f_{i}$ is the $\mathrm{i}$ th component of the body force vector and $u_{i}$ is the $\mathrm{i}$ th component of the displacement vector; for a linear Hookean elastic solid the $C_{i j k l}$ encapsulate the constitutive stress-strain relation and the Einstein summation notation is assumed. The elastic wave scattering problem can thus be seen as that of solving the partial differential equation (2) with boundary conditions (3) or (4) [21]. Alternatively, solutions can be found by deriving the material response to singular sources, that is Green's functions, and treating the scatterer as a superposition of secondary sources using Huygens' principle and far-field radiation conditions [22]. In either case, because of the competing dimensions involved, full analytical solutions exist only for a small class of defect geometries with only regular shapes such as a sphere or an infinite circular cylinder studied over the entire frequency range of interest. Therefore, from the beginning of the subject, approximate solution methods were of interest. An intuitive progression is to consider solving separately for the wave field satisfying just the wave equation, and one that also 
satisfies the boundary conditions at an obstacle: relating these two separate local solutions then leads to the global solution; the first hybrid models for elastic wave scattering problems such as the method of matched asymptotic expansion, introduced for elastodynamics [23]-[25] in the 1970's used this breakdown of the field.

Although this allows one to approach scattering involving a wider class of defect shapes such as spheroids or ellipsoids, analytic matched asymptotic approaches are fundamentally limited to low-frequencies; the need to study ever more complex scatterers meant that other techniques were required to represent the vicinity of the defect. The rapid rise of computational capabilities in the last two decades also allowed for the possibility of using purely numerical methods. A literature survey shows that in dealing with these challenges similar hybrid modelling methods have since been developed across a range of areas where elastic wave scattering is employed as a sensing tool [25]-[30]. However, the hybrid models used in the present work are perhaps closest in their intent and form, to the global-local method originating in the structural mechanics community and the domain reduction method from the field of geophysics.

Originally proposed [31] in the 1970's, the global-local method involves a two-step solution procedure, where a global solution is first found using variational (e.g., Rayleigh-Ritz) methods and the results are fed into the local area model represented by standard FE analysis. The technique was extended to study fusing two numerical methods [32] and has found extensive application in aircraft structural analysis and in the fracture mechanics of composites [33]-[35]. In the last decade the method also found application in ultrasonic NDE research as a powerful way of studying large scale problems especially in multilayer and other waveguide scattering problems: these methods involve mode matching at the boundary of the local region, which is modelled using numerical methods such as FE or the Boundary Element method [36], [37].

Complementary to the scattering problems created by complex defect geometries or topologies geophysicists are also concerned with representing large propagation distances and constructing realistic three-dimensional models. Thus several researchers (see for example [38]-[40]) proposed a two-step analysis that would involve a rapid computation of global fields for a simplified material, combined with a more involved analysis of a small local volume enclosing defects, complex material or topography. This work has culminated in the FE-FD coupling procedure for three-dimensional problems called the domain reduction method [38] that has recently become very popular [41] for studying fully three-dimensional problems.

Despite the diverse origins, and the surprisingly vast number of practical implementation schemes, reported in the literature all such hybrid methods share a formalism that is not often stated explicitly. In a very recent paper set in the context of geophysics, Oprsal et al [42] have elegantly summarized, as well as unified, developments with these varied fields, under a simple and rigorous mathematical framework. In the following section, we follow their approach to set out the fundamental basis for and justification behind using hybrid models for elastodynamic problems. This exercise will also help in appreciating the nuances, capabilities and limitations of domain-reduction hybrid models. However, in preparation for the developments later on in this paper, we describe the formalism in terms of source-solutions rather than as direct solutions to the partial differential equations in (2). 


\section{GENERALIZING THE HYBRID MODELLING PROCEDURE}

\section{A. Formalism and hybrid modelling paradigm}

Let $P_{T}$ be the total problem containing the excitation sources and the defective region and posed in a linear elastic medium. Conventionally, this entire problem would be studied using a single method through its entirety, either analytical or numerical. As discussed in Section II, hybrid models instead solve separately the material response to applied excitation, and the response of a scatterer to the incident wave field, and then connect them to yield the solution to the total problem. Stated formally, thus hybrid models as illustrated in Figure 2(a) and 2(b), propose to solve two auxiliary problems $P_{G}$ and $P_{L}$, that together yield the total result.

In the first problem $P_{G}$ as shown in Figure 2(a), the medium is idealized to be defect-free and with the same material properties as the actual problem. The incident field field ${ }^{\text {incident }}=\left\{\boldsymbol{u}^{i n}, \boldsymbol{\sigma}^{i n}\right\}$ is then computed as the response of the resulting medium to loading conditions. In the application of interest here the incident field is generally a pulse of finite duration. However, for certain aspects of the method it is convenient to perform a Fourier transform with respect to time and to consider the individual time-harmonic components of the signal, later recombining them for the time-dependent result.

For time-harmonic motion it is assumed that the time-variation of all the variables is proportional to $\exp (+\mathrm{i} \omega t)$, a factor which is suppressed henceforth. Using an integral representation theorem [43] and retaining the same origin and coordinate system as for the total problem as shown in Figure 2(a), this incident field is given by:

$$
\begin{gathered}
u_{k}^{i n}(\boldsymbol{r})=\int_{C_{S}}\left[\sigma_{i j}^{\text {load }}(\boldsymbol{s})\right) G_{i ; k}(\boldsymbol{r} \mid \boldsymbol{s}) \\
-\Sigma_{i j ; k}\left(\left((\boldsymbol{r} \mid \boldsymbol{s}) u_{i}^{\text {load }}(\boldsymbol{s})\right)\right] \cdot n_{j} \mathrm{~d} S, \quad \boldsymbol{r} \in V_{+}
\end{gathered}
$$

where the integral is taken around the contour $C_{S}$, enclosing the source, shown in Figure $2(\mathrm{a}) ; G_{k ; i}(\boldsymbol{p} \mid \boldsymbol{r})$ is the component of the second rank Green's displacement tensor giving the displacement component in the $\hat{e}_{k}$ direction at position $\boldsymbol{p}$ due to a unit point force $f_{i}=\delta(\boldsymbol{p}-\boldsymbol{r}) \hat{e}_{i}$ applied in the $\hat{e}_{i}$ direction at $\boldsymbol{r}$ and $\Sigma_{k j ; i}(\boldsymbol{p} \mid \boldsymbol{r})$ is the corresponding component of the third rank Green's stress tensor; both $\boldsymbol{G}$ and $\boldsymbol{\Sigma}$ are symmetric in $\boldsymbol{p}$ and $\boldsymbol{r}$. The three-dimensional delta function $\delta(\boldsymbol{p}-\boldsymbol{r})$ has the property:

$$
\int_{V_{+}}\left[\delta\left((\boldsymbol{p}-\boldsymbol{r}) \hat{e}_{i}\right) u_{k}(\boldsymbol{p})\right] \mathrm{d} V= \begin{cases}u_{k}(\boldsymbol{r}), & \boldsymbol{r} \in V_{+} \\ 0, & \text { otherwise }\end{cases}
$$

where $V_{+}$is the unbounded region outside the loading region. $n_{j}$ are the components of inward normal to an arbitrary contour $C_{S}$ suited to the calculation of the material response to the loading conditions.

Realistic loading conditions are usually complicated and equation (5) is not evaluated analytically. Let us assume that the incident field is obtained using some solution procedure $M_{1}$. The material response field ${ }^{\text {incident }}=$ $\left\{\boldsymbol{u}^{i n}, \boldsymbol{\sigma}^{i n}\right\}$ is computed everywhere - in particular within the small region $R_{2}$ enclosing the defective region in the original problem.

In the second problem $P_{L}$ shown in Figure 2(b), field quantities obtained from $P_{G}$ act as excitation on the boundaries of scatterers, leading to the response field ${ }^{\text {scattered }}=\left\{\boldsymbol{u}^{s c}, \boldsymbol{\sigma}^{s c}\right\}$. In general the defective region could 
contain material, as well as geometric discontinuities, acting as scatterers, but for simplicity, here we assume that it only contains a finite number $N$ of the latter. Again, the scattered field is expressed using the representation theorem,

$$
\begin{gathered}
u_{k}^{s c}(\boldsymbol{r})=\int_{C_{D}}\left[\alpha(\boldsymbol{s}) \sigma_{i j}^{i n}(\boldsymbol{s}) G_{i ; k}(\boldsymbol{r} \mid \boldsymbol{s})\right. \\
\left.-\Sigma_{i j ; k}\left((\boldsymbol{r} \mid \boldsymbol{s}) \alpha(\boldsymbol{s}) u_{i}^{i n}(\boldsymbol{s})\right)\right] \cdot n_{j} \mathrm{~d} S, \quad \boldsymbol{r} \in V_{+}
\end{gathered}
$$

as in Achenbach [44] where $\alpha(\boldsymbol{s})$ now are general scattering coefficients and the contour $C_{D}=\sum_{m=1}^{N} S_{m}$ is the sum of all surfaces of the individual scatterers.

Eq. (7) is also usually not solved analytically and a method $M_{2}$ is chosen, that is more accurate in the vicinity of the scatterers, to solve the problem.

In the final step, the linear behaviour of the medium is invoked and the total field $\left\{\boldsymbol{u}^{\text {tot }}, \boldsymbol{\sigma}^{\text {tot }}\right\}$ is obtained as a linear superposition of the two computed fields:

$$
\text { field }^{\text {total }}=\text { field }^{\text {incident }}+\text { field }^{\text {scattered }}
$$

On the boundary $\beta_{2}$ of the defective region in the total problem, this step also ensures the continuity of the wave fields thus avoiding spurious reflections:

$$
u_{i}^{\text {tot }}\left(\beta_{2}^{-}\right)=u_{i}^{i n}\left(\beta_{2}\right)+u_{i}^{s c}\left(\beta_{2}^{+}\right)
$$

where $\beta_{2}^{-}, \beta_{2}^{+}$denote the regions immediately inside and outside $\beta_{2}$ respectively. Linearity means the tractions can also be represented as a sum:

$$
T_{i}^{t o t}\left(\beta_{2}^{-}\right)=T_{i}^{i n}\left(\beta_{2}\right)+T_{i}^{s c}\left(\beta_{2}^{+}\right) .
$$

It is instructive to examine the steps involved in the scheme to gain crucial insight into the validity, capabilities and limitations of the procedure. Firstly, the superposition of wave fields as in Eq. (8), and also in Eqs. $(9,10)$, are only achieved if the material response is linear. The use of linearity implicitly renders the boundary $\beta_{2}$ permeable for the scattered field, instead of itself becoming a cause of further scattering. These hybrid models can have the properties of the defect domain non-elastic, as long as they retain their linear behaviour. Scatterers with non-linear behaviour can also be studied as long as they are located away from the boundaries. However, the hybrid model as derived here cannot treat non-linear media in general and for such applications, alternative formulations must be found [42].

Finally, though it may not be immediately apparent, it is the step in Eq. (8) that ties hybrid models to specific applications. The summation in Eq. (8) requires that the scattered field be known over the whole of the original model space. However, because the calculation in Eq. (7) must be performed to high accuracy, it is only economical to use the method $M_{2}$ chosen for this purpose, to compute the scattered field within the small region $\beta_{2}$ enclosing 
the defective region. Therefore some special method must be devised to then obtain the scattered field elsewhere, which then, is inevitably limited to the two solution procedures $M_{1}$ and $M_{2}$. We will look into a scheme to overcome this issue in the next section.

\section{B. Generalized procedure}

Examination of Eq.s (5) and (7) reveals a scheme to generalize this method, as illustrated in Figure 2(c) and 2(d). The excitation and defect regions are assumed, respectively, to be located within fictitious regions $R_{1}$ and $R_{2}$ of the original total problem to be studied. We take the response of the region $R_{1}$, to the applied loading, to be calculated using the method $M_{1}$, giving the incident field generated by the sources within region $R_{1}$,

$$
\text { field }^{\text {incident }}(\boldsymbol{s})\left|\boldsymbol{s} \in R_{1}=\boldsymbol{R e s p o n s e}\left(R_{1}\right)\right|_{M_{1}}^{\text {Applied loading }}
$$

Now, assuming that the bulk material has a known response, and thus knowing this incident field on the boundary $\beta_{1}$, we can express its value elsewhere using the representation theorem,

$$
\begin{gathered}
u_{k}^{i n}(\boldsymbol{r})=\int_{\beta_{1}}\left[\sigma_{i j}^{i n}(\boldsymbol{s}) G_{i ; k}(\boldsymbol{r} \mid \boldsymbol{s})\right. \\
\left.-\Sigma_{i j ; k}\left((\boldsymbol{r} \mid \boldsymbol{s}) u_{i}^{i n}(\boldsymbol{s})\right)\right] \cdot n_{j} \mathrm{~d} S, \quad \boldsymbol{r} \in V_{+} .
\end{gathered}
$$

Next let the response of the region $R_{2}$ to the incident field be obtained using the method $M_{2}$, yielding the scattered field,

$$
\text { field }\left.^{\text {scattered }}(\boldsymbol{s})\right|_{\boldsymbol{s} \in R_{2}}=\boldsymbol{R} \text { esponse }\left.\left(R_{2}\right)\right|_{M_{2}} ^{\text {Incident field }}
$$

Using this information on the boundary $\beta_{2}$ then, we can obtain the global scattered field using the known material response again,

$$
\begin{gathered}
u_{k}^{s c}(\boldsymbol{r})=\int_{\beta_{2}}\left[\sigma_{i j}^{s c}(\boldsymbol{s}) G_{i ; k}(\boldsymbol{r} \mid \boldsymbol{s})\right. \\
\left.-\Sigma_{i j ; k}\left((\boldsymbol{r} \mid \boldsymbol{s}) u_{i}^{s c}(\boldsymbol{s})\right)\right] \cdot n_{j} \mathrm{~d} S, \quad \boldsymbol{r} \in V_{+} .
\end{gathered}
$$

The response of the medium to the incident field and the scattered field, separated according to (8) in this way, as the local response to the applied loading in the first region and as secondary sources within the second (defect) region, respectively, can be viewed as a general wave propagator interface between the two model domains:

$$
\text { field }^{\text {global }}=\left.\boldsymbol{R e s p o n s e}\left(R-R_{1}-R_{2}\right)\right|_{M_{1} / M_{2}} ^{\text {Incident/ scattered field }}
$$

where $R-R_{1}-R_{2}$ is the idealized medium obtained by excluding both $R_{1}$ and $R_{2}$ from the total region of study, $R$. This approach, in which the field in each local region is calculated separately, is appropriate to applications in NDE because the interest there is in the propagation and scattering of finite-duration pulses, such that the behaviour in each region can be separated in time. This scheme can also handle multiple scattering domains, as long as the 
event is separable in time. On the other hand, if the interest is to model simultaneous coupled behaviour in the multiple regions, then a modification of this procedure to a fully-coupled form would be necessary; this is outside the scope of this paper.

Since the contours $\beta_{1}$ and $\beta_{2}$ will be chosen to be simple ones, we can evaluate Eqs (15) in a straightforward manner. Coded up in a convenient manner, they can then be used to interface any two methods $M_{1}$ and $M_{2}$ in a customised hybrid model.

Thus the total problem is split into two local ones (11) and (13), and the local fields so obtained are globalized using the material response, as in Eq (15). The total solution is then again obtained using the linear superposition described in Eq. (8). This is the gist of the generalization procedure proposed here.

In the following section, an example of a generic wave propagator module is considered for the common problem of the propagation of ultrasonic waves in the bulk of a homogeneous isotropic medium.

\section{A GENERIC WAVE PROPAGATOR}

\section{A. Scheme}

We consider ultrasonic phenomena in the bulk of a homogeneous isotropic medium, which is a common abstraction for a large class of ultrasonic wave inspection problems. The physics of this abstraction also permits analysis by in-plane elasticity. We begin with the assumption that the potentials for the field generated by the sources, together with their normal gradients, are known through some mechanism on the boundary $\beta_{1}$ of region $R_{1}$. The use of potentials provides generality since they can be used to conveniently obtain any of the different field quantities such as displacements, velocities, stresses or tractions used primarily in various commercial packages. We take the field propagated from region 1 as the incident field on region 2, and vice versa. The propagator integrals can then be written as, for example,

$$
\begin{gathered}
\phi^{i n}\left(\boldsymbol{r}_{2}\right)=\int_{\beta_{1}}\left[\phi^{i n}\left(\boldsymbol{r}_{1}\right) \nabla_{1} G_{\phi}\left(\boldsymbol{r}_{2} \mid \boldsymbol{r}_{1}\right)\right. \\
\left.-G_{\phi}\left(\boldsymbol{r}_{2} \mid \boldsymbol{r}_{1}\right) \nabla_{1} \phi^{i n}\left(\boldsymbol{r}_{1}\right)\right] \cdot \boldsymbol{n} \mathrm{d} S \\
\psi^{i n}\left(\boldsymbol{r}_{2}\right)=\int_{\beta_{1}}\left[\psi^{i n}\left(\boldsymbol{r}_{1}\right) \nabla_{1} G_{\psi}\left(\boldsymbol{r}_{2} \mid \boldsymbol{r}_{1}\right)\right. \\
\left.-G_{\psi}\left(\boldsymbol{r}_{2} \mid \boldsymbol{r}_{1}\right) \nabla_{1} \psi^{i n}\left(\boldsymbol{r}_{1}\right)\right] \cdot \boldsymbol{n} \mathrm{d} S,
\end{gathered}
$$

where the subscript $i, i=1,2$ refers to points on the boundary $\beta_{i}$ of region $R_{i}$ and $\phi$ and $\psi$ are compressive and shear potentials, respectively, from which the displacements are calculated as

$$
\begin{aligned}
& u_{x}=\frac{\partial \phi}{\partial x}+\frac{\partial \psi}{\partial y} \\
& u_{y}=\frac{\partial \phi}{\partial y}-\frac{\partial \psi}{\partial x}
\end{aligned}
$$

The potentials satisfy the homogeneous Helmholtz equations

$$
\left(\nabla^{2}+\omega^{2} \gamma^{2}\right) \phi=0
$$


and

$$
\left(\nabla^{2}+\omega^{2}\right) \psi=0
$$

in which $\gamma$ is the ratio of the shear wave speed to the compressional wave speed in the elastic medium. The corresponding Greens functions satisfy the inhomogeneous equations

$$
\left(\nabla_{1}^{2}+\omega^{2} \gamma^{2}\right) G_{\phi}\left(\boldsymbol{r}_{2} \mid \boldsymbol{r}_{1}\right)=\delta\left(\boldsymbol{r}_{2}-\boldsymbol{r}_{1}\right)
$$

and

$$
\left(\nabla_{1}^{2}+\omega^{2}\right) G_{\psi}\left(\boldsymbol{r}_{2} \mid \boldsymbol{r}_{1}\right)=\delta\left(\boldsymbol{r}_{2}-\boldsymbol{r}_{1}\right)
$$

whose outgoing wave solutions are known to be

$$
\begin{aligned}
G_{\phi}\left(\boldsymbol{r}_{2} \mid \boldsymbol{r}_{1}\right) & =-\frac{\mathrm{i}}{4} \mathrm{H}_{0}^{(2)}\left(\gamma \omega\left|\boldsymbol{r}_{2}-\boldsymbol{r}_{1}\right|\right), \\
G_{\psi}\left(\boldsymbol{r}_{2} \mid \boldsymbol{r}_{1}\right) & =-\frac{\mathrm{i}}{4} \mathrm{H}_{0}^{(2)}\left(\omega\left|\boldsymbol{r}_{2}-\boldsymbol{r}_{1}\right|\right),
\end{aligned}
$$

and $\mathrm{H}_{0}^{(2)}$ is the zeroth order Hankel function of the second kind which ensures outgoing waves. These are straightforward to evaluate numerically and allow a straightforward numerical evaluation of the integrals of equations (16) and (17). Thus the incident potentials on and near to $\beta_{2}$ may be calculated. If required, the normal derivatives of the potentials may be approximated numerically on the second boundary from values of the potential near to the boundary. Applying these values as input to another suitable (defect domain) model allows the potentials, $\phi_{s}^{s c}$ and $\psi_{s}^{s c}$, due to scattering of this field in region 2 to be obtained, along with their normal derivatives, on the boundary $\beta_{2}$ and used in a similar way to calculate the scattered field elsewhere, which may itself be used as an incident field on another scattering region. For example the incident field for secondary scattering in region 1 would be calculated as:

$$
\begin{gathered}
\phi_{s}^{i n}\left(\boldsymbol{r}_{1}\right)=\int_{\beta_{2}}\left[\phi_{s}^{s c}\left(\boldsymbol{r}_{2}\right) \nabla_{2} G_{\phi}\left(\boldsymbol{r}_{1} \mid \boldsymbol{r}_{2}\right)\right. \\
\left.-G_{\phi}\left(\boldsymbol{r}_{1} \mid \boldsymbol{r}_{2}\right) \nabla_{2} \phi_{s}^{s c}\left(\boldsymbol{r}_{2}\right)\right] \cdot \boldsymbol{n} \mathrm{d} S, \\
\psi_{s}^{i n}\left(\boldsymbol{r}_{1}\right)=\int_{\beta_{2}}\left[\psi_{s}^{s c}\left(\boldsymbol{r}_{2}\right) \nabla_{2} G_{\psi}\left(\boldsymbol{r}_{1} \mid \boldsymbol{r}_{2}\right)\right. \\
\left.-G_{\psi}\left(\boldsymbol{r}_{1} \mid \boldsymbol{r}_{2}\right) \nabla_{2} \psi_{s}^{s c}\left(\boldsymbol{r}_{1}\right)\right] \cdot \boldsymbol{n} \mathrm{d} S .
\end{gathered}
$$

\section{B. Adaptation to cases defined by displacements and stresses}

We next adapt this scheme to a scenario where displacements and stresses are assumed to be the typical output in the methods $M_{1}$ and $M_{2}$ used to represent the wave propagation and scattering model domains; this form of output occurs in a fairly large number of numerical schemes particularly in commercial codes. Thus stresses and displacements are known on the contours $\beta_{1}$ and $\beta_{2}$ and the goal is to express the potentials, and their normal derivatives, to be input to the wave propagator integrals, in terms of these values: here we describe an efficient scheme for this calculation. For ease of exposition here the contours are assumed to be rectangles with sides parallel to the $x-$ and $y-$ axes, and dimensionless variables are utilised. 
In dimensionless variables and assuming no body-force, the equations of motion (1) may be written for timeharmonic in-plane elastic motion as

$$
\begin{aligned}
-\omega^{2} u_{x} & =\frac{\partial \sigma_{x x}}{\partial x}+\frac{\partial \sigma_{x y}}{\partial y} \\
-\omega^{2} u_{y} & =\frac{\partial \sigma_{x y}}{\partial x}+\frac{\partial \sigma_{y y}}{\partial y}, \\
\sigma_{x x} & =\gamma^{-2} \frac{\partial u_{x}}{\partial x}+\left(\gamma^{-2}-2\right) \frac{\partial u_{y}}{\partial y}, \\
\sigma_{x y} & =\frac{\partial u_{y}}{\partial x}+\frac{\partial u_{x}}{\partial y} \\
\sigma_{y y} & =\left(\gamma^{-2}-2\right) \frac{\partial u_{x}}{\partial x}+\gamma^{-2} \frac{\partial u_{y}}{\partial y},
\end{aligned}
$$

where $\sigma_{x x}, \sigma_{x y}$ and $\sigma_{y y}$ are the stress components, $u_{x}$ and $u_{y}$ are displacement components. In terms of notation, we use $x, y$ and $x_{1}, x_{2}$ interchangeably.

Differentiating (18) with respect to $x$, and (19) with respect to $y$, and adding the equations shows that

$$
\nabla^{2} \phi=\frac{\partial u_{x}}{\partial x}+\frac{\partial u_{y}}{\partial y}
$$

which, combined with (20) gives an expression for $\phi$ :

$$
\phi=\frac{-1}{\omega^{2} \gamma^{2}}\left(\frac{\partial u_{x}}{\partial x}+\frac{\partial u_{y}}{\partial y}\right) .
$$

Another expression for $\partial u_{x} / \partial x+\partial u_{y} / \partial y$ is obtained by adding (30) and (32):

$$
\sigma_{x x}+\sigma_{y y}=2\left(\gamma^{-2}-1\right)\left(\frac{\partial u_{x}}{\partial x}+\frac{\partial u_{y}}{\partial y}\right) .
$$

Thus, the required expression for $\phi$ in terms of the stress is

$$
\phi=\frac{\left(\sigma_{x x}+\sigma_{y y}\right)}{2 \omega^{2}\left(\gamma^{2}-1\right)} .
$$

Similarly, $\psi$ is obtained by differentiating (18) with respect to $y$ and (19) with respect to $x$ and subtracting them to give

$$
\nabla^{2} \psi=\frac{\partial u_{x}}{\partial y}-\frac{\partial u_{y}}{\partial x}
$$

and hence, using the Helmholtz equation (21)

$$
\psi=\frac{-1}{\omega^{2}}\left(\frac{\partial u_{x}}{\partial y}-\frac{\partial u_{y}}{\partial x}\right)
$$

These terms occur only in the stress definition equation (31) hence both terms cannot be simultaneously eliminated. However, as the displacements are known on the boundary, their tangential derivatives may be calculated numerically there. Hence, these expressions for $\psi$ may be used on rectangular boundaries:

$$
\begin{array}{ll}
\psi=\frac{-1}{\omega^{2}}\left(\sigma_{x y}-2 \frac{\partial u_{y}}{\partial x}\right) & \text { on } y=\text { constant } \\
\psi=\frac{-1}{\omega^{2}}\left(2 \frac{\partial u_{x}}{\partial y}-\sigma_{x y}\right) & \text { on } x=\text { constant. }
\end{array}
$$


On the parts of the boundary with $y=$ constant, the normal derivative is $\partial / \partial y$, but the required expressions may only contain derivatives with respect to $x$. Equation (34) may be differentiated with respect to $y$, and then, in turn, (32) used to eliminate $\partial u_{y} / \partial y$, (29) to eliminate $\partial \sigma_{y y} / \partial y$, and (31) used to eliminate $\partial u_{x} / \partial y$, as

$$
\frac{\partial \phi}{\partial y}=\frac{-1}{\omega^{2}}\left(\frac{\partial \sigma_{x y}}{\partial x}-2 \frac{\partial^{2} u_{y}}{\partial x^{2}}-\omega^{2} u_{y}\right) .
$$

Similarly, differentiating (38) with respect to $y$, using (31) to eliminate $\partial u_{x} / \partial y$, (28) to eliminate $\partial \sigma_{x y} / \partial y$ and (30) and (32) to eliminate $\partial u_{y} / \partial y$, results in

$$
\frac{\partial \psi}{\partial y}=\frac{1}{\omega^{2}}\left(\omega^{2} u_{x}+\frac{1}{2\left(1-\gamma^{2}\right)} \frac{\partial \sigma_{x x}}{\partial x}+\frac{1}{2\left(1-\gamma^{2}\right)} \frac{\partial \sigma_{y y}}{\partial x}\right) .
$$

On the parts of the boundary with $x=$ constant, the normal derivative is $\partial / \partial x$, but the required expressions may only contain derivatives with respect to $y$. Hence, differentiating (34) with respect to $x$ and then using (30) to eliminate $\partial u_{x} / \partial x$, (28) to eliminate $\partial \sigma_{x x} / \partial x$ and then (31) to eliminate $\partial u_{y} / \partial x$ results in

$$
\frac{\partial \phi}{\partial x}=\frac{-1}{\omega^{2}}\left(\frac{\partial \sigma_{x y}}{\partial y}-2 \frac{\partial^{2} u_{x}}{\partial y^{2}}-\omega^{2} u_{x}\right) .
$$

Similarly, differentiating (38) with respect to $x$ and then using (31) to eliminate $\partial u_{y} / \partial x$, (29) to eliminate $\partial \sigma_{x y} / \partial x$ and then (30) and (32) to eliminate $\partial u_{x} / \partial x$ results in

$$
\frac{\partial \psi}{\partial x}=\frac{-1}{\omega^{2}}\left(\omega^{2} u_{y}+\frac{1}{2\left(1-\gamma^{2}\right)} \frac{\partial \sigma_{x x}}{\partial y}+\frac{1}{2\left(1-\gamma^{2}\right)} \frac{\partial \sigma_{y y}}{\partial y}\right) .
$$

These expressions then enter propagator integrals such as $(16,17)$ resulting in the values of the potentials on the second boundary, thereby allowing calculation of the physical quantities there such as stress and displacement, to be used in the forcing for that subproblem. Subsequently these expressions may be applied to the scattered stress and displacement fields exiting the defect region to obtain scattered potentials on that boundary for use in the propagator integrals $(26,27)$ in order to obtain the potentials and hence the stresses and displacements in the observation region.

\section{VALIDATION}

The wave propagator adaptation, as described in Section IV.B, is designed to be a generic hybrid modelling interface between any two of a large class of numerical, or analytical, modelling procedures for two-dimensional bulk elastic wave phenomena.

We now validate this procedure using a prototype hybrid model where the regions $R_{1}$ and $R_{2}$ are both represented by a single method, chosen to be the FE method. The hybrid interface was coded up as a MATLAB function and for simplicity both the contours $\beta_{1}$ and $\beta_{2}$ are chosen to be rectangles. The interface code considers as input, the stresses and displacements on $\beta_{1}$ surrounding the source, and predicts a required quantity (stress or displacement) on $\beta_{2}$ surrounding a required destination position. The prototype hybrid model, together with the functions of the hybrid interface, is illustrated in Figure 3.

Sections V.A and V.B consider the forward wave propagation and the reverse wave scattering problems, respectively. In both sections, the approach taken is that first the total problem is modelled by a single FE model of the 
whole system and the results so obtained are then compared with predictions by the example hybrid interface. The excitation consists of a mixed-point force [45], [46] applied in one direction at a single node, leading to circular crested waves; both Longitudinal $(\mathrm{P})$ and Shear $(\mathrm{S})$ waves are considered. The basic code works in the frequency domain, but for the completely time-domain calculations required for most NDE applications, a simple frequency loop enables recovery of time-domain predictions through the use of Fourier Transforms, as demonstration in the final section. The FE simulations are performed by a time-marching scheme in the time-domain. Thus forward and inverse Fourier Transforms are used as part of the calculations. The time-domain field quantities delivered by the FE simulation in the source domain are transformed to the frequency domain by a fast Fourier Transform (FFT), for input to the hybrid interface. Then, on arrival at the destination domain, the output field quantities from the hybrid interface are transformed to the time-domain by inverse FFT.

Finally, Section V.C presents the hybrid solution of the forward and the backward problems consisting of physically-separated domains with an example of real practical relevance. This is the scattering of a wave pulse from a row of three Side-Drilled Holes (SDHs). SDHs are used routinely in practical inspections for calibration of equipment. Thus calibration blocks containing SDHs are readily available and familiar to practitioners, and the prediction of scattering from them is a useful and relevant demonstration.

\section{A. Forward wave propagation}

We consider the propagation of ultrasound in the bulk of a defect-free infinite isotropic elastic medium. This will be represented using the two modelling approaches: the full FE model of the entire domain, and the prototype hybrid model linking two smaller FE model domains for the wave generation and the forward propagation locations respectively. We use the same geometry for both of these approaches, as illustrated in Figure 4(a). The full FE model is represented by the entire region of the figure. The analysis of this provides us with reference results for the validation of the hybrid model. The hybrid model links the two smaller FE domains (or "boxes") which are shown by the two red squares, representing $\beta_{1}$ and $\beta_{2}$. The domain on the left surrounds the source while the domain on the right is the destination. The explicit time commercial FE package ABAQUS (version 6.7) [5] is used in the simulations; a central objective of our approach is to develop a versatile scheme that is not model or package dependent and so can be used with standard, easily available, commercial codes, and we believe this to be a key contribution of this work.

The bulk of the defect-free infinite medium of the full FE model is represented by a two-dimensional plane-strain domain bounded at its edges by absorbing layers with increasing damping [12]. The black rectangle surrounding the full FE model indicates the boundary between the elastic and absorbing parts of the model. The size of the total domain, including the absorbing regions, is $1.5 \mathrm{~m}$ by $0.58 \mathrm{~m}$ and that of the actual area of study is $1.14 \mathrm{~m}$ by $0.22 \mathrm{~m}$. Uniform linear quadratic square elements with the material properties of Aluminium (Elastic modulus 71

GPa, density $2700 \mathrm{~kg} / \mathrm{m}^{3}$ and Poisson ratio 0.33) are used to mesh the whole domain. The choice of material is arbitrary, aluminium is chosen for convenience, and similar results and trends occur for other isotropic materials.

The simulation is run with time-domain excitation provided by a 5-cycle Hanning windowed toneburst [47] 
centred at the required frequency and applied as a force in a single direction ( $x$ or $y$ ), at a single node. In the example in Figure 4(a), the force is applied in the location indicated as 'Source', in the $y$ direction. Compressional, $\mathrm{P}$, and shear, $\mathrm{S}$, waves propagate primarily perpendicular and parallel to the excitation direction respectively, and are eventually absorbed at the model edges. Two separate cases are chosen, with different excitations (direction of applied force and frequency of the applied signal) to drive primarily S- or P-waves, respectively, to propagate into the destination box. For the S-wave case the nodal point-force is applied in the $y$ direction, while for the P-wave case, it is applied in the $x$ direction. The purpose of this is that, with the destination box positioned as shown in Figure 4(a), we detect primarily S-waves when the nodal point-force is applied in the $y$ direction, and primarily $\mathrm{P}$-waves when the force is applied in the $x$ direction. The element size at $1 \mathrm{~mm}$ is the same in both cases, but an appropriate centre-frequency (100 kHz and $200 \mathrm{kHz}$ respectively for S- and P-wave studies), is chosen to ensure a constant mesh density of about 32 elements per centre-wavelength, $\lambda_{c}$; this is the wavelength at the centre-frequency of the wave. This ensures that there is a sufficient number of elements per wavelength for accurate numerical modelling of the elastic wave propagation [47]. In both studies, the simulation was run up to a time when S- and P-waves respectively had travelled completely across the destination box. The colour contours in Figure 4(a) present snapshots of the total displacement magnitude, obtained from the simulation for the S-wave case, showing the wave field at two selected times during propagation.

Displacements and stresses are obtained along the two $100 \mathrm{~mm}$ (approximately $3.2 \lambda_{c}$ long) boxes, one surrounding the source and the other bounding an arbitrary forward destination located about 26 wavelengths from it. It must be mentioned that while the displacements can be monitored directly at the boundary nodes, ABAQUS outputs stresshistories only as elemental quantities. Therefore, stresses were obtained on two concentric layers bounding the nodal contour from the inside and the outside respectively, and then these were averaged to give the (approximate) stresses at the nodal locations. Field quantities so obtained on the source box are then transformed into the frequency domain and the values at the centre-frequency are extracted. These are fed into the hybrid interface code along with material and geometrical details to obtain predictions for their values on the destination box. These values are then compared with those from the full FE simulations obtained at the boundary of the destination box.

Results for the $\mathrm{S}$ and $\mathrm{P}$ wave cases are shown in Figure 5: the comparison is along nodes constituting the 'Left' boundary of the monitored destination box which is indicated by a row of white dots in Figure 4(a). The Y axis in Figure 5(a)-(d) is scaled with respect to the maximum amplitude of the incident field at the source location of the full FE model. In both S and P wave cases, we observe excellent agreement between the hybrid prediction and the full-FE results.

There are some minor differences. The errors averaged over the 100 nodal points are shown in Table 1 . One small source of error is the averaging of stresses at the centroids of elements surrounding a node; this is necessary because the stresses are calculated at element centroids whereas the displacements are calculated at nodes. Another source of error comes from the fact that the accuracy with which the frequency spectrum is extracted depends on the number of points in the time-signal at the source box. This in turn, depends on the number of time-increments used in the FE simulation and subsequently, the number of increments at which the output field is recorded. In 
addition, spatial discretisation inevitably introduces errors to the result to an extent. For the full FE and the hybrid simulations, approximately $\lambda_{c} / 30$ is selected as the element size since it is well below the typical limit used for accurate modelling (for example [47]), but the accuracy might be still improved with a more dense mesh. However, despite these minor issues the agreement is very satisfying.

\section{B. Back-scattering}

Next we consider the back-scattering of waves from a reflector. For simplicity we start with a very basic scatterer consisting of a straight rigid barrier located within the bulk of an infinite isotropic medium. Again we use the same geometry for both a single FE model of the complete domain and the hybrid procedure using two smaller domains; the results are compared in a similar manner. The full-FE model set up is identical to that of the previous section V.A, except for the fact that the destination box now contains the barrier; we use a rectangular box for convenience, but the method works in principle for any shape. The barrier was realized by selecting nodes along a required straight line and fixing all displacements on them to be identically zero for the duration of the simulation. The barrier was placed centrally in the destination box (Figure 4). Again, two separate cases were set up for when S- and P-waves, respectively, primarily interact with the barrier, whose nominal physical length is kept constant at $64 \mathrm{~mm}$. The different centre-frequency used for the excitation signal ensured that in both models, the barrier's effective length also remained constant at $2 \lambda_{c}$. This choice of barrier-length is significant as it has a bearing upon how the signals obtained on the boundaries of the destination box are processed to obtain the input for the reverse hybrid process.

The generic hybrid interface developed in Section IV.B requires as input, the purely scattered field, without any incident signal components. This can be extracted from the total field at the destination box by two processing methods: we could allow for the dimensions of the box to be large so that the incident and scattered components are separated in time, or we could subtract out the incident component from the total signal (see [13] for a more detailed discussion of these two processing approaches). The separation method usually requires larger dimensions than the subtraction method, which theoretically just requires a very small destination box for the FE calculation of the total field. However a very small destination box is also likely to be located in the near-field of the scattered waves. To illustrate this better, let us assume that the destination contour is a circle instead of a square, and that the scatterer too, is of an ideal, circular shape, as shown inset within the plot in Figure 6. We then define the radius of the contour required for achieving signal separation as the separation-radius and similarly a near-field-radius, estimates for which can be obtained respectively from time-of-flight analysis and piston-behaviour of the scatterer (aperture formula $a^{2} /(4 \lambda)$ where $a$ is the aperture-length). Figure 6 then presents these two radii plotted against the scatterer radius for the case where the excitation signal consists of a 5-cycle long Hanning windowed toneburst. We observe that the separation method always requires a very large dimension of the monitoring contour. Thus our implementation using the subtraction method provides significant advantage.

An advantage of the hybrid propagator interface, as developed in this paper, is that it can handle field quantities even within the near-field efficiently: therefore the destination box bounding the defective region can be made small. 
We thus note that, as can be seen from Figure 6, the $2 \lambda_{c}$ long barrier means that the boundaries of the $3.2 \lambda_{c}$ long destination box, especially the top and the bottom ones, lie close to or within the expected near-field of the barrier. The dimensions are, of course, far lower than those required for the separation of incident and scattered signals.

The full-FE simulations were run up to a time such that S- and P-waves respectively interacted with the barrier and scattered back, as shown for example, using the contour of the total displacement magnitude obtained from the S-wave study, in Figure 4(b). Displacements and stresses were again extracted in the frequency domain on the boundary of the destination box. Frequency-domain field quantities monitored at this same location from studies in the previous section V.A without the defect were then subtracted from these values. Field quantities now contain only the scattered component and form the input to the reverse hybrid interface, to obtain predictions for the field at a required backscatter location.

Figure 7 shows the comparison for the S- and P- wave studies, for a backscattered position forming the 'Right' nodes of the original source box (indicated in Figure 4(b)) and their averaged errors are shown in Table 1. Again, we observe excellent agreement between the hybrid prediction and the full-FE result. The remarks on averaging of stresses and number of time-points in the FE simulation made at the end of section V.A are also pertinent for this case. Furthermore, for accurate performance of the inverse hybrid interface, it is important that the incident signals are obtained at the same nodal positions as the total scattered signals, so that there are no spurious signals in the subtracted purely scattered signals.

\section{Full forward and backscattered example}

In the previous sections, hybrid calculations were demonstrated and verified separately for propagating and scattering waves. However, the benefit of the hybrid method is realised when the whole model is physically divided into separate domains and these are used to simulate the full forward propagation and back-scattering problem. The source and scatterer can then be arbitrarily distant from each other with no computational cost penalty. Such an approach is demonstrated here. As an example, we take up a case from a realistic NDE procedure, namely that of the scattering of ultrasound from a row of side-drilled holes. The physical case to be modelled was a solid block of steel with three parallel circular holes, as shown in Figure 8(a). It is common for NDE practitioners to use blocks with SDHs to perform calibration of ultrasound instrumentation, such that signal amplitudes can be known when interpreting reflections from un-seen defects in real inspections. In our case we choose to model the signals which are sent and received by a transducer placed on the left side of the sample. The incident wave is generated by the transducer, and after it has scattered from the holes, the returning signal is monitored at the same location representing the transducer as a receiver.

Figure 8(b) shows a Full FE model which was used to simulate the whole problem in the conventional manner, and in this case to provide reference results for validation of the hybrid calculations. The full FE domain including source and three SDHs has dimensions $55 \times 56 \mathrm{~mm}^{2}$. The main domain is $19 \times 20 \mathrm{~mm}^{2}$ surrounded by an absorbing region simulating an infinite medium. Three SDHs of $1 \mathrm{~mm}$ radius are aligned with $6 \mathrm{~mm}$ intervals. Sources representing a transducer are located $4 \lambda_{c}$ from the centre of the middle hole, perpendicular to a line 
connecting the centres of the three holes. The $10 \mathrm{~mm}$ transducer is modelled by 101 nodal points excited by a synchronised input signal. The $\mathrm{S}$ wave is chosen, so $y$ direction forces are applied to the sources. The chosen signal is a $1 \mathrm{MHz}$ centre frequency, five-cycle toneburst signal. Figure 9 shows a snapshot of the FE domain with the wave scattering from the defect. Inside the domain shown in the figure is free-meshed with nominal $0.1 \mathrm{~mm}$ element size, in order to verify the hybrid method in a general case with irregular defects, but the rest of the model including the absorbing regions is regularly meshed.

Next this problem is modelled using the hybrid approach, the domains for which are illustrated in Figure 8(c). The source domain is selected to be $42 \times 52 \mathrm{~mm}^{2}$ regularly meshed, its size excluding absorbing region is $8 \times 16$ $\mathrm{mm}^{2}$. The source is placed at the centre of the domain, and half $\lambda_{c}$ away from the source is the Source Monitoring Box (SMB). The defect domain of $45.5 \times 56 \mathrm{~mm}^{2}$ with three SDHs has a free-meshed region of $9.5 \times 20 \mathrm{~mm}^{2}$ and is regularly meshed in the absorbing region. The Defect Monitoring Box (DMB) is located at half $\lambda_{c}$ from the holes, and an excitation line is located approximately half $\lambda_{c}$ in front of the DMB.

The full simulation was started by a forward hybrid calculation. The model of the source domain (Figure 8(c)) was run to obtain the outgoing signal at the SMB. The hybrid calculation was then used to predict the propagation of the signal to the defect domain, expressing the arriving signal by tractions along the line shown in the figure as the excitation line. Calculations were then performed in the defect domain, using these tractions as the input. Two cases were run, one including the SDHs, and the other without, so that the scattered field could be found by subtracting the latter from the former. Finally the scattered field in the defect domain was monitored at the DMB, and the hybrid calculation was used once more to predict the signal arriving back at the source.

Figure 10 shows snapshots of the two domains with the wave propagating from the source to the defect domain. Wave generation can be seen in Figure 10(a). The wave signal then arrives, via the hybrid model and its input at the excitation line, in the defect domain, and can be seen propagating in the positive $\mathrm{x}$ direction in Figure 10(b). Waves also propagate from here in the negative direction, but are absorbed in the absorbing region to the left of the excitation line. Figure 10(c) shows the waves in the defect domain being scattered by the SDHs. They are then monitored on the DMB, in order to predict the field returning to the source (receiver). The wave field in Figure 10(c), although complex, can be seen to match very closely to the wave field in the full FE model at the same moment in time, which was shown in Figure 9.

Figure 11 shows a comparison of the results for the full FE model and the two hybrid models. The results which are shown, by way of example, are for the averaged y component of the displacement over the location of the transducer (location shown in Figure 8). In both cases the signal is chosen, arbitrarily, to have an amplitude of unity at the source location. Figure 11(a) shows the time-domain signal, that is, a simulation of the signal which would be received by the transducer. Figure 11(b) shows the same information transformed to the frequency-domain. Excellent agreement between the two cases can be seen. 
For a more detailed comparison, Figure 12 shows only the scattered waves in the time interval 5 - $20 \mu$ sec. The amplitudes of the scattered signal in Figure 12(a) is seen to be about $20 \%$ of that of the incident signal. Despite the complex fields around the 3 SDHs shown in Figures 9 and 10(c), the shape of the scattered signal in Figure 12(a) is simple, because this signal is dominated by the reflection from the centre hole; excellent agreement between the results for the full FE model and those for the the hybrid model can be seen. The frequency domain signal in Figure 12(b) also agrees well within the bandwidth 0.6-1.4 MHz, but there are noticeable differences below and above that range; these result from the fact that the hybrid calculations only used frequencies to cover that bandwidth. In addition, there are some differences due to the reasons mentioned in Section V-A. However, overall the results show excellent agreement.

The CPU times for the full FE and the hybrid method are approximately 21 and 23 minutes, respectively, in an identical computation system. The similarity of times is to be expected given the broadly similar model sizes. The size of the full FE model is $3080 \mathrm{~mm}^{2}$ while that of the source and the defect domains for the hybrid calculation are $2184 \mathrm{~mm}^{2}$ and $2548 \mathrm{~mm}^{2}$. Therefore superficially the hybrid approach does not present a computational advantage. However, this is because of our choice of an example problem for which the scatterer is close to the source, which was made deliberately in order to limit the computer resources needed for the full FE model. The real advantage of the hybrid model, and indeed its purpose, will be for cases in which the wave propagation path is much longer, so that it becomes prohibitively expensive, or indeed impossible, to perform the full FE calculation. This advantage will be amplified further in future when the method is extended to three dimensions.

\section{CONCLUSIONS}

In this paper we have developed a general modelling tool to simulate practical ultrasonic inspection. Due to the ever increasing sophistication of practical inspection, it is best to develop this in a modular manner for each step, such as wave generation, propagation, scattering and post-processing, and then integrate the different steps for practical inspection. In this context hybrid modelling schemes display maximal advantage: a suitable scheme must be generic and independent of the constituent modelling techniques. Although hybrid modelling methods are commonly known in the literature and may be as old as the study of elastic wave scattering itself [20], most developments (including recent ones, such as [3] for efficient FE models and [48] where a CIVA-FE link is being pursued) are tied to specific sets of modelling techniques. We show how the limitation of the hybrid schemes to particular modelling techniques arises out of their fundamental formulation and give a formalism to generalize them. We generate a generic hybrid modelling interface by considering the case of bulk ultrasonic wave phenomena, an abstraction common to a large class of practical inspection problems. This interface works within a prototype hybrid model consisting of two smaller FE model-domains, and the feasibility of such an approach is demonstrated for bulk ultrasonic wave propagation and scattering examples. Sources of error and ways of improving the accuracy of the interface are also discussed.

\section{ACKNOWLEDGMENT}

The authors would like to thank Rolls-Royce Plc, Derby, U.K, for their support through this research effort. 


\section{REFERENCES}

[1] E. Le Clézio, M. Castaings, and B. Hosten, "The interaction of the S0 Lamb mode with vertical cracks in an aluminium plate," Ultrasonics, vol. 40, pp. 187-192, 2002.

[2] M. Frehner, S. M. Schmalholz, E. H. Saenger, and H. Steeb, "Comparison of finite difference and finite element methods for simulating two-dimensional scattering of elastic waves," Physics of the Earth and Planetary Interiors, vol. 171, pp. 112-121, 2008.

[3] P. D. Wilcox and A. Velichko, "Efficient frequency-domain finite element modeling of two-dimensional elastodynamic scattering," $J$. Acoust. Soc. Am., vol. 127, 2010.

[4] M. Castaings, C. Bacon, B. Hosten, and M. V. Predoi, "Finite element predictions for the dynamic response of thermo-viscoelastic material structures," J. Acoust. Soc. Am., vol. 115, pp. 1125-1133, 2004.

[5] ABAQUS v6.7 Analysis User's Manual, 2007, http://www.simulia.com/.

[6] PZFlex, Weidlinger Associates, Inc. 399 West El Camino Real Mountain View, CA 94040-2607, USA, http://www.pzflex.com/contact.aspx.

[7] COMSOL User's Guide version 3.2, 2006, http://www.comsol.com/.

[8] J.-P. Berenger, "A perfectly matched layer for the absorption of electromagnetic waves," J. Comp. Phys., vol. 114, pp. $185-200,1994$.

[9] E. A. Skelton, S. D. M. Adams, and R. V. Craster, "Guided elastic waves and perfectly matched layers," Wave Motion, vol. 44, pp. 573-592, 2007.

[10] M. Castaings and C. Bacon, "Finite element modeling of torsional wave modes along pipes with absorbing materials," J. Acoust. Soc. Am., vol. 119, pp. 3741-3751, 2006.

[11] J. D. De Basabe and M. K. Sen, "Grid dispersion and stability criteria of some common finite-element methods for acoustic and elastic wave equations," Geophysics, vol. 72, pp. T81-T95, 2007.

[12] M. Drozdz, E. Skelton, R. V. Craster, and M. J. S. Lowe, "Modeling bulk and guided wave propagation in unbounded elastic media using absorbing layers in commercial FE packages," in Review of Progress in Quantitative NDE, D. O. Thompson and D. E. Chimenti, Eds., vol. 26. New York: American Institute of Physics, 2007, pp. 87-94.

[13] P. Rajagopal, M. Drozdz, and M. J. S. Lowe, "Towards improved finite element modeling of the interaction of elastic waves with complex defect geometries," in Review of Progress in Quantitative NDE, D.O.Thompson and D. E. Chimenti, Eds., vol. 28. American Institute Physics, 2009, pp. 49-56.

[14] P. Calmon, S. Mahaut, S. Chatillon, and R. Raillon, "CIVA: An expertise platform for simulation and processing NDT data," Ultrasonics, vol. 44, pp. e975-e979, 2006.

[15] S. K. Datta, "Diffraction of plane elastic waves by ellipsoidal inclusions," J. Acoust. Soc. Am., vol. 61, pp. 1432-1437, 1977.

[16] R. Paskaramoorthy, A. H. Shah, and S. K. Datta, "Scattering of flexural waves by a crack in a plate," Engng. Frac. Mech., vol. 33, pp. 589-598, 1989.

[17] J. Zhu and A. H. Shah, "A hybrid method for transient wave scattering by flaws in composite plates," Intl. J. Solids Struc., vol. 34, pp. 1719-1734, 1997.

[18] Z. Chang and A. Mal, "Scattering of Lamb waves from a rivet hole with edge cracks," Mechanics of Materials, vol. 31, pp. 197-204, 1999.

[19] R. Lerch, H. Landes, H. Kaarmann. "Finite Element Modeling of the Pulse-Echo Behavior of Ultrasound Transducers," Proceedings of the IEEE Ultrasonics Symposium, 1021-1025 Cannes: IEEE. 1994

[20] R. P. Kanwal, "Theory of diffraction and matched asymptotic expansions," J. Math. Phys., vol. 8, pp. 821-822, 1967.

[21] Y. H. Pao and C. C. Mow, Diffraction of Elastic Waves and Dynamic Stress Concentrations. New York: Crane Russak, 1973.

[22] V. Varatharajulu, "Reciprocity relations and forward amplitude theorems for elastic waves," J. Math. Phys., vol. 18, pp. 537-543, 1977.

[23] S. K. Datta, C. Fortunko, and R. King, "Sizing of surface cracks in a plate using SH waves," IEEE Ultrasonics Symposium, pp. 863-867, 1981.

[24] A. Lakhtakia, V. V. Varadan, and V. K. Varadan, "Reflection characteristics of an elastic slab containing a periodic array of elastic cylinders: SH wave analysis," J. Acoust. Soc. Am., vol. 80, pp. 311-316, 1986.

[25] A. Lakhtakia, V. V. Varadan, and V. K. Varadan, "Reflection characteristics of an elastic slab containing a periodic array of circular elastic cylinders: P and SV wave analysis," J. Acoust. Soc. Am., vol. 83, pp. 1267-1275, 1988.

[26] I. D. Abrahams and G. R. Wickham, "Scattering of elastic-waves by a small inclined surface-breaking crack," J. Mech. Phys. Solids, vol. 40, pp. 1707-1733, 1992. 
[27] I. D. Abrahams and G. R. Wickham, "Scattering of elastic-waves by an arbitrary small imperfection in the surface of a half-space," J. Mech. Phys. Solids, vol. 40, pp. 1683-1706, 1992.

[28] S. W. Liu and S. K. Datta, “Scattering of ultrasonic wave by cracks in a plate,” J. Appl. Mech., vol. 60, pp. 352-357, 1993.

[29] X.-M. Wang, C. F. Ying, and M. X. Li, "Scattering of antiplane shear waves by a circular cylinder in a traction-free plate," J. Acoust. Soc. Am., vol. 108, pp. 913-923, 2000.

[30] H. Bai, A. H. Shah., N. Popplewell, and S. K. Datta, "Scattering of guided waves by circumferential cracks in steel pipes," J. Appl. Mech., vol. 68, pp. 619-631, 2001.

[31] C. D. Mote Jr., “Global-local finite element,” Int. J. Num. Meth. Engng., vol. 3, pp. 565-574, 1971.

[32] O. C. Zienkiewicz, D. W. Kelly, and P. Bettess, "The coupling of the finite element method and boundary solution procedures," Int. J. Num. Meth. Engng., vol. 11, pp. 355-375, 1977.

[33] J. B. Ransom and N. F. Knight, "Global-local stress-analysis of composite panels,” Comp. Struct., vol. 37, pp. 375-395, 1990.

[34] S. R. Voleti, N. Chandra, and J. R. Miller, "Global local analysis of large-scale composite structures using finite element methods," Comp. Struct., vol. 58, pp. 453-464, 1996.

[35] R. K. Kapania, "Global/local analysis of composite plates with cutouts,” Comp. Mech., vol. 19, pp. 386-396, 1997.

[36] A. Mal and Z. S. Chang, "A semi-numerical method for elastic wave scattering calculations," Geophys. J. Int., vol. 143, pp. 328-334, 2000 .

[37] Y. Cho and J. L. Rose, "An elastodynamic hybrid boundary element study for elastic guided wave interactions with a surface breaking defect," Intl. J. Solids Struc., vol. 37, pp. 4103-4124, 2000.

[38] J. Bielak and P. Christiano, "On the effective seismic input for non-linear soil-structure interaction systems," Earthquake Engineering and Structural Dynamics, vol. 12, pp. 107-119, 1984.

[39] D. Fah, P. Suhadolc, S. Mueller, and G. F. Panza, "A hybrid method for the estimation of ground motion in sedimentary basins: Quantitative modeling for Mexico city," Bull. Seism. Soc. Am., vol. 84, pp. 383-399, 1994.

[40] J. Zahradnk and P. Moczo, "Hybrid seismic modeling based on discrete-wave number and finite-difference methods," Pure Appl. Geophys., vol. 148, pp. 21-38, 1996.

[41] S. Kontoe, L. Zdravkovic, and D. Potts, "The domain reduction method for dynamic coupled consolidation problems in geotechnical engineering," International Journal for Numerical and Analytical Methods in Geomechanics, vol. 32, pp. 659-680, 2008.

[42] I. Oprsal, C. Matyska, and K. Irikura, "The source-box wave propagation hybrid methods: general formulation and implementation," Geophys. J. Int., vol. 176, pp. 555-564, 2009.

[43] J. D. Achenbach, A. K. Gautesen, and H. McMaken, Ray methods for waves in elastic solids. London: Pitman, 1982.

[44] J. D. Achenbach, Reciprocity in elastodynamics. Cambridge University Press, 2003.

[45] P. Wilcox, "Modeling the excitation of Lamb and SH waves by point and line sources," in Review of Progress in Quantitative NDE, D.O.Thompson and D. E. Chimenti, Eds., vol. 23. American Institute Physics, 2004, pp. $206-213$.

[46] P. Rajagopal and M. Lowe, "Short range scattering of the fundamental shear horizontal guided wave mode normally incident at a throughthickness crack in an isotropic plate," J. Acoust. Soc. Am., vol. 122, pp. 1527-1538, 2007.

[47] D. N. Alleyne and P. Cawley, "The interaction of Lamb waves with defects," IEEE Transactions on Ultrasonics, Ferroelectrics and Frequency control, vol. 39, pp. 381-397, 1992.

[48] M. Darmon, N. Leymarie, S. Chatillon, and S. Mahaut, "Modelling of scattering of ultrasounds by flaws for NDT," in Ultrasonic wave propagation in nonhomogeneous media, ser. Springer proceedings in Physics. Springer, 2009, pp. 61-71. 
Figure \& Table captions

Fig. 1. Illustration of the various modules that constitute a typical ultrasonic inspection simulation.

Fig. 2. (a) and (b) show the Global and Local problems, respectively, in traditional Hybrid models. (c) and (d) show the Global and Local problems in the proposed generalization of the Hybrid modelling method.

Fig. 3. Illustration of the prototypical hybrid model, together with the functions of the hybrid interface.

Fig. 4. Snapshots of the contour of total displacement magnitude from a FE simulation, (a) for the S-wave propagating towards the destination square and (b) for the S-wave case with a rigid barrier in the destination box showing the wave field just after scattering from the barrier. The full FE model is represented by the complete area shown; the two red boxes are the small domains selected to be modelled by the hybrid approach. Thus the hybrid model results can be compared directly with the full model results.

Fig. 5. Comparison of the hybrid interface model to full FE simulations for the forward propagation case. (a) $\mathrm{x}$ displacements and (b) y displacements for S-wave propagation; (c) $\mathrm{x}$ displacements and (d) y displacements for P-wave propagation. The comparison is performed along all the nodes on the left boundary of the destination box, at the centre frequency of the signal in each case.

Fig. 6. Separation and near-field radius plotted versus the defect radius for the case where the excitation signal consists of a 5-cycle Hanning windowed toneburst. For a $2 \lambda_{c}$ long defect, the domain radius would need to be at least $3.5 \lambda_{c}$ to ensure separation of incident and scattered signals, and at least $1 \lambda_{c}$ to escape the near-field. Inset: an idealised circular domain with a circular defect.

Fig. 7. Comparison of the hybrid interface model to full FE simulations for the back-scattering case. (a) $x$ displacements and (b) y displacements for S-wave propagation; (c) x displacements and (d) y displacements for P-wave propagation. The comparison is performed along all the nodes on the right boundary of the source box, at the centre frequency of the signal in each case.

Fig. 8. Realistic example of prediction of scattering from a row of side drilled holes (SDHs). (a) illustration of test block with three side-drilled holes; a transducer is placed on the left of the block and used to generate the test signal and receive the back-scattered signal. Illustration of the model of this case, showing (b) full FE domain and (c) the two boxes for the hybrid model.

Fig. 9. Snapshot of propagating wave through the two physically separated domains. (a) Source domain at 6 $\mu$ sec: propagating waves are monitored at the Source Monitoring Box (SMB) as input to the hybrid model which then predicts the signals at the destination box. (b) Defect domain without the SDHs at $8 \mu$ sec: propagating waves are generated by imposing tractions on the excitation line, representing the signal coming from the source box. For clarity, the field is shown for the case without the presence of SDHs. (c) Defect domain with the SDHs at $8 \mu$ sec: The scattered waves are monitored at the DMB and fed into the hybrid interface to predict the signal back at the source location (now the receiver).

Fig. 10. Snapshot of propagating wave through the two physically separated domains. (a) Source domain at 6 $\mu$ sec: propagating waves are monitored at the Source Monitoring Box (SMB) and used to calculate signals on 
secondary sources. (b) Defect domain without the SDHs at $8 \mu$ sec: propagating waves are generated by imposing traction on the excitation line, representing the signal coming from the source box. For clarity, the field is shown for the case without the presence of SDHs. (c) Defect domain with the SDHs at $8 \mu \mathrm{sec}$ : The scattered waves are monitored at the DMB and fed into the hybrid interface to predict the signal at the receiver.

Fig. 11. Comparison of predicted amplitudes of the average y displacement at the nodes on the receiver, monitoring the waves scattering back from the side-drilled holes. Predictions of full FE (solid), back scattering only (dash-dot), and forward-backward (dashed) models in (a) time-domain; (b) frequency-domain.

Fig. 12. Comparison as in Fig.11: Identical plots but showing only the scattered wave, and the data from 5 to $20 \mu \mathrm{sec}$ inside the box in Fig.11a.

Table 1. Errors averaged over 100 nodal points for each of the figure 5(a)-(d) and 7(a)-(b) 


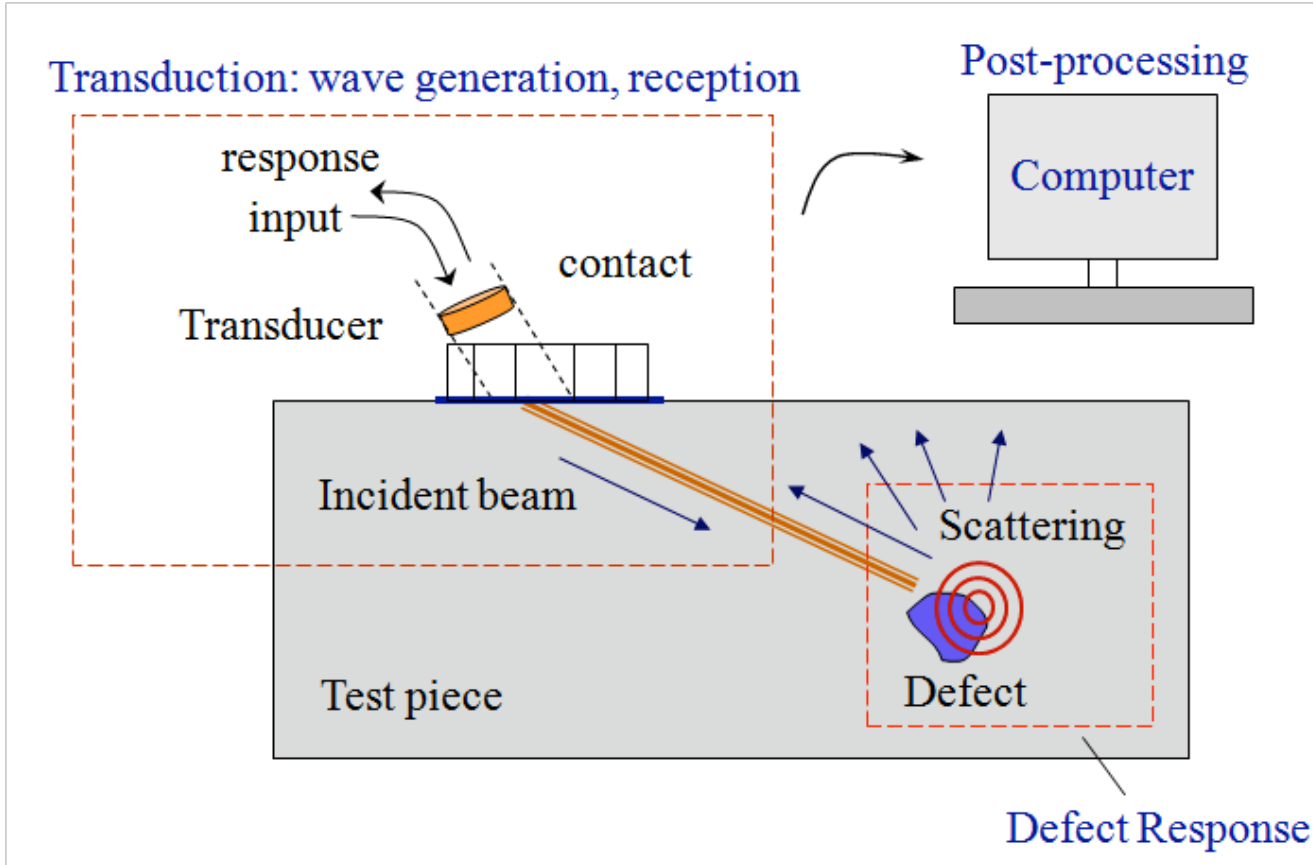

Fig. 1. Illustration of the various modules that constitute a typical ultrasonic inspection simulation. 
(a)

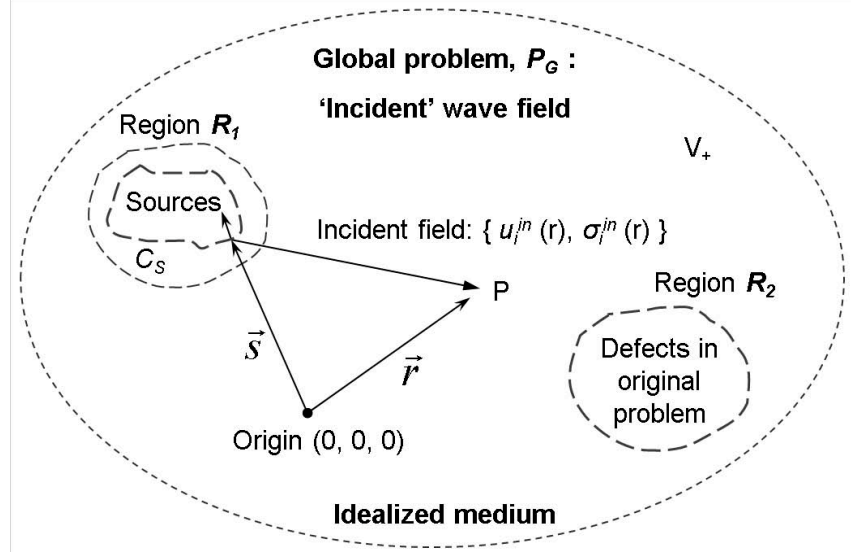

(c)

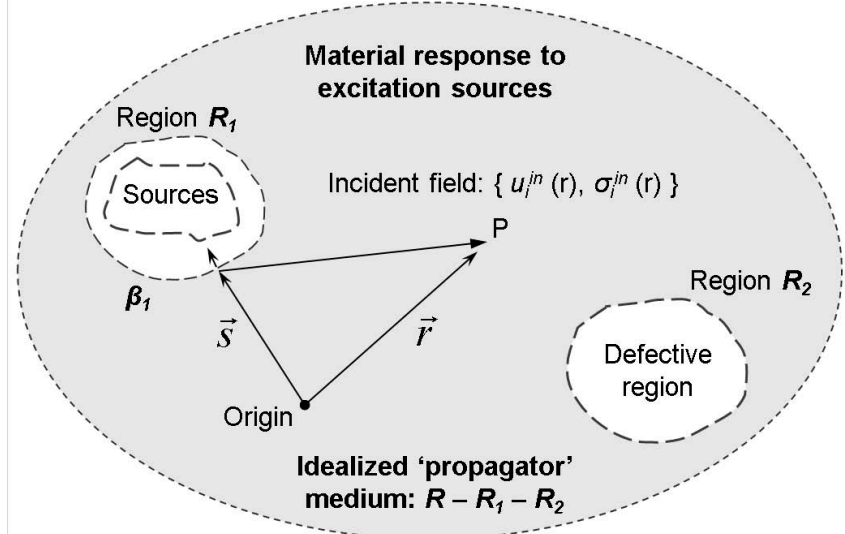

(b)

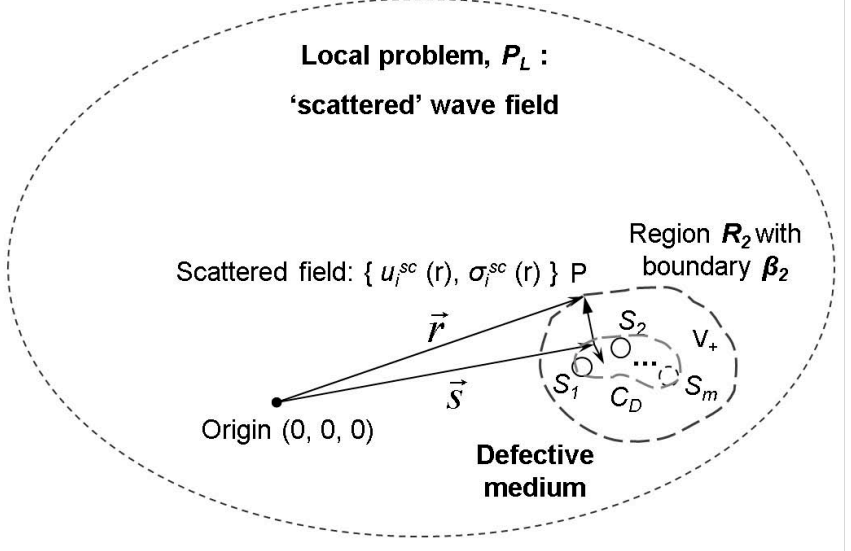

(d)

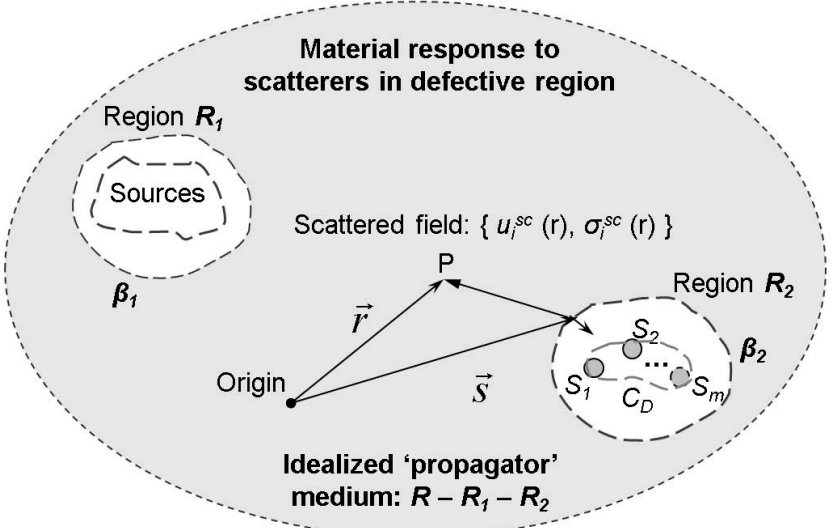

Fig. 2. (a) and (b) show the Global and Local problems, respectively, in traditional Hybrid models. (c) and (d) show the Global and Local problems in the proposed generalization of the Hybrid modelling method. 


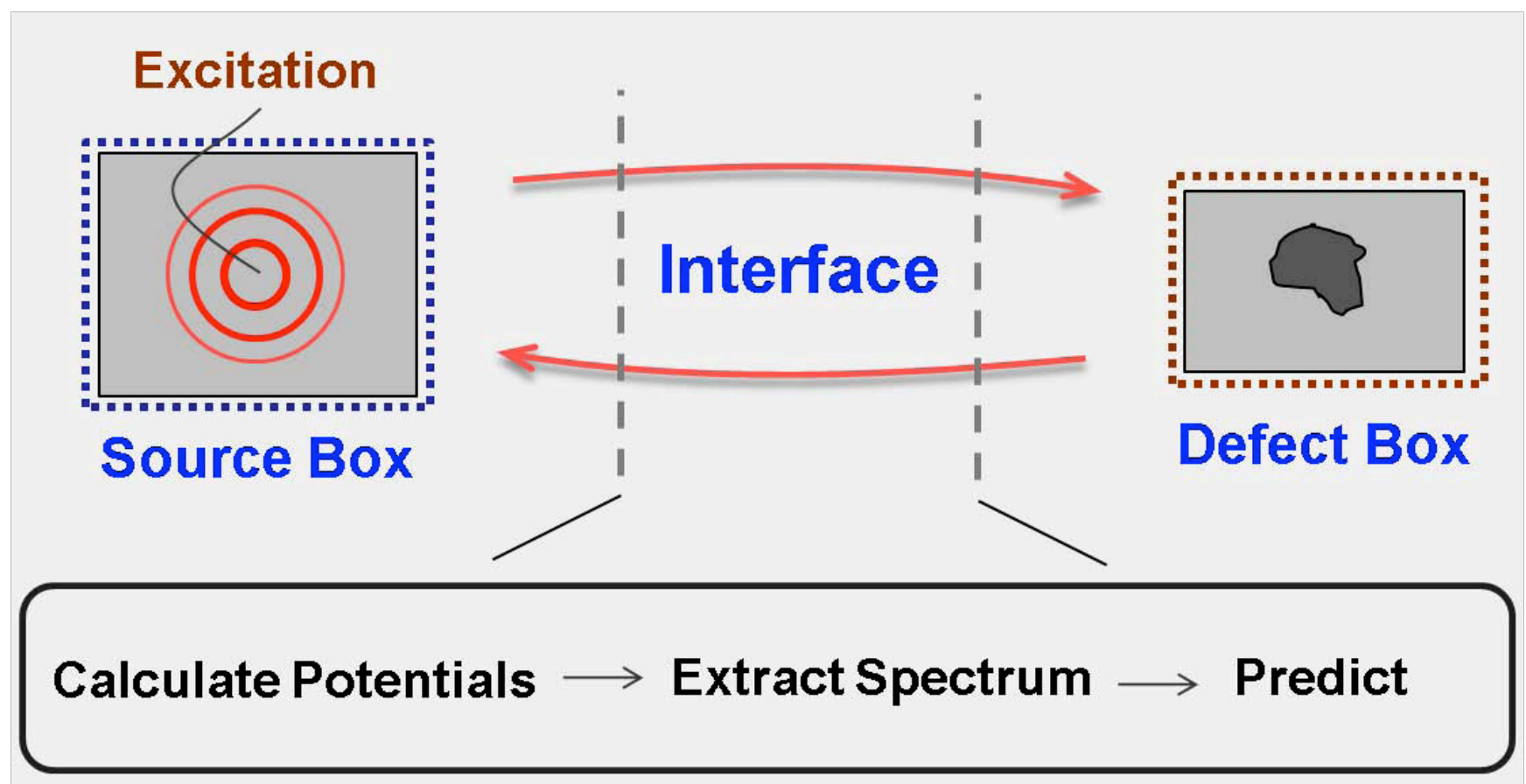

Fig. 3. Illustration of the prototypical hybrid model, together with the functions of the hybrid interface. 


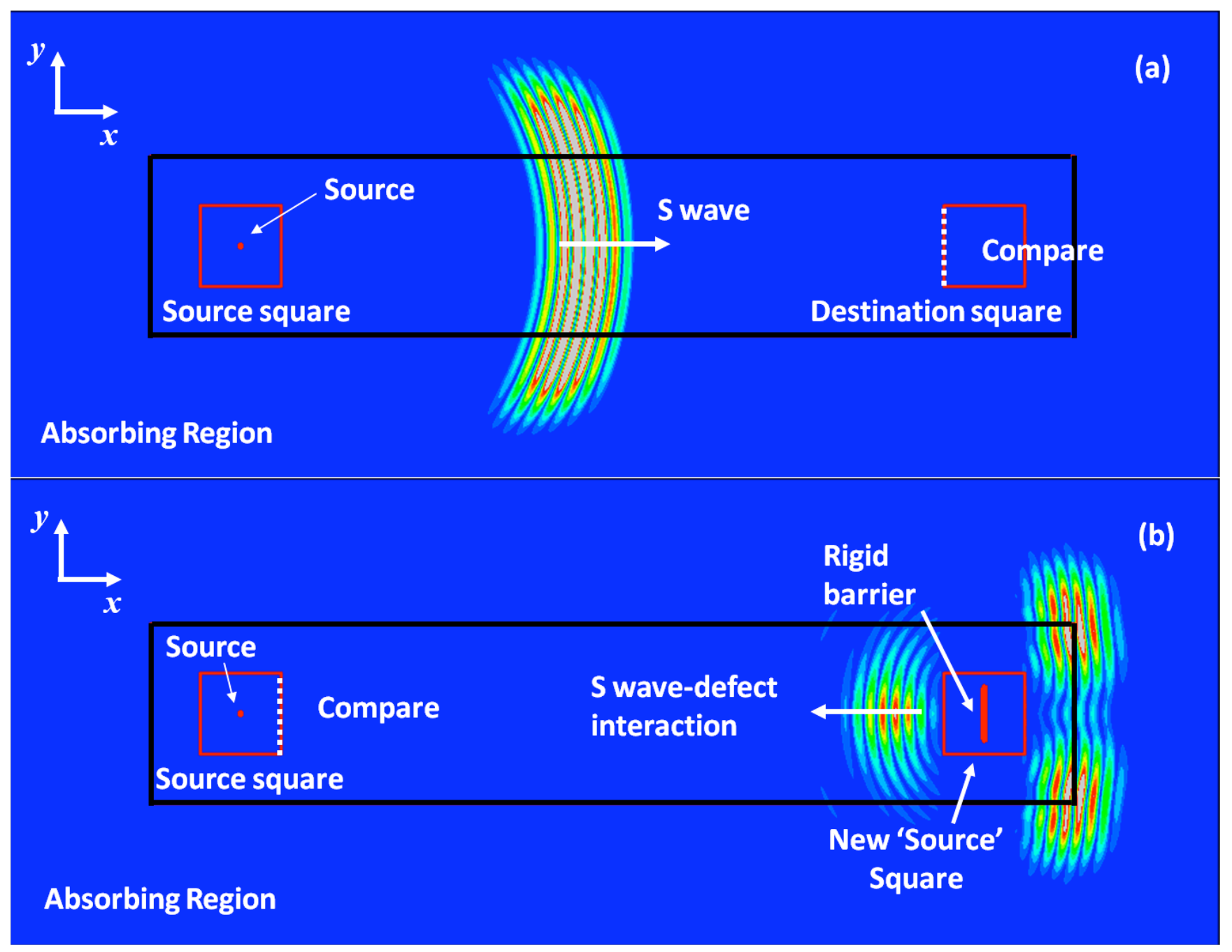

Fig. 4. Snapshots of the contour of total displacement magnitude from a FE simulation, (a) for the Swave propagating towards the destination square and (b) for the S-wave case with a rigid barrier in the destination box showing wave field just after scattering from the barrier. The full FE model is represented by the complete area shown; the two red boxes are the small domains selected to be modelled by the hybrid approach. Thus the hybrid model results can be compared directly with the full model results. 

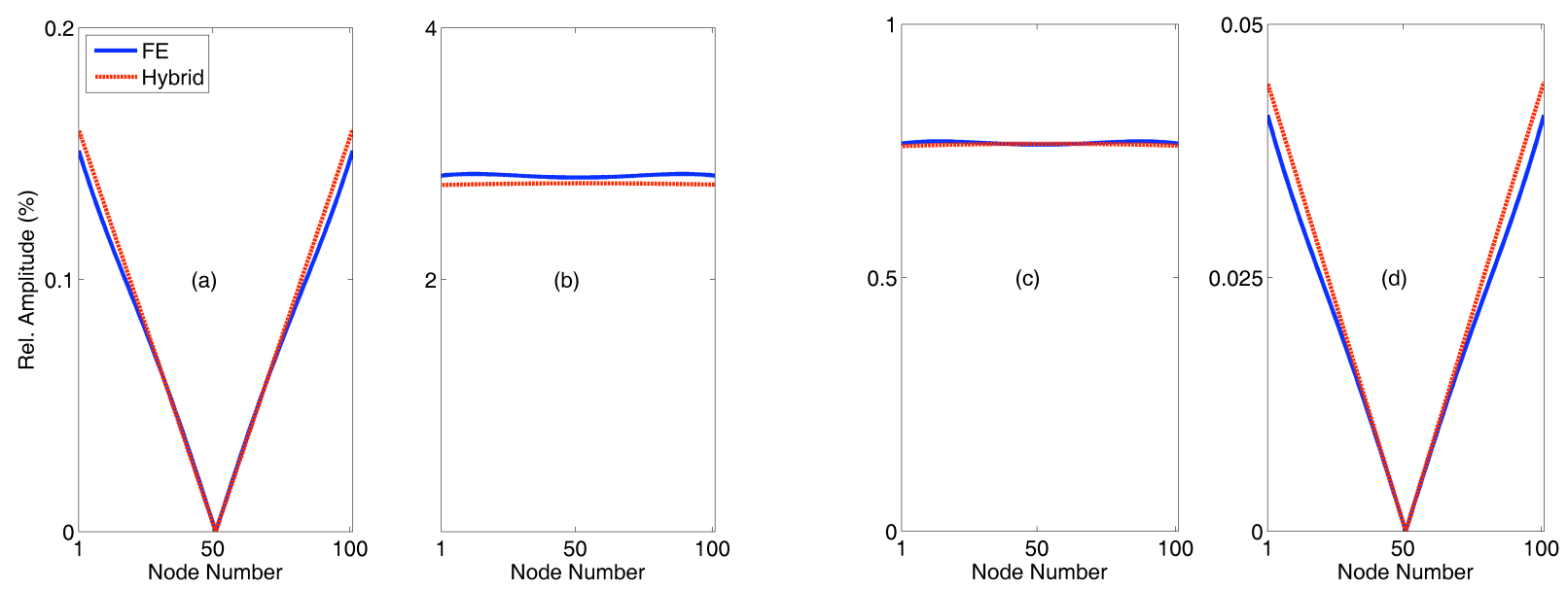

Fig. 5. Comparison of the hybrid interface model to full FE simulations for the forward propagation case. (a) $x$ displacements and (b) y displacements for S-wave propagation; (c) $x$ displacements and (d) y displacements for P-wave propagation. The comparison is performed along all the nodes on the left boundary of the destination box, at the centre frequency of the signal in each case. 


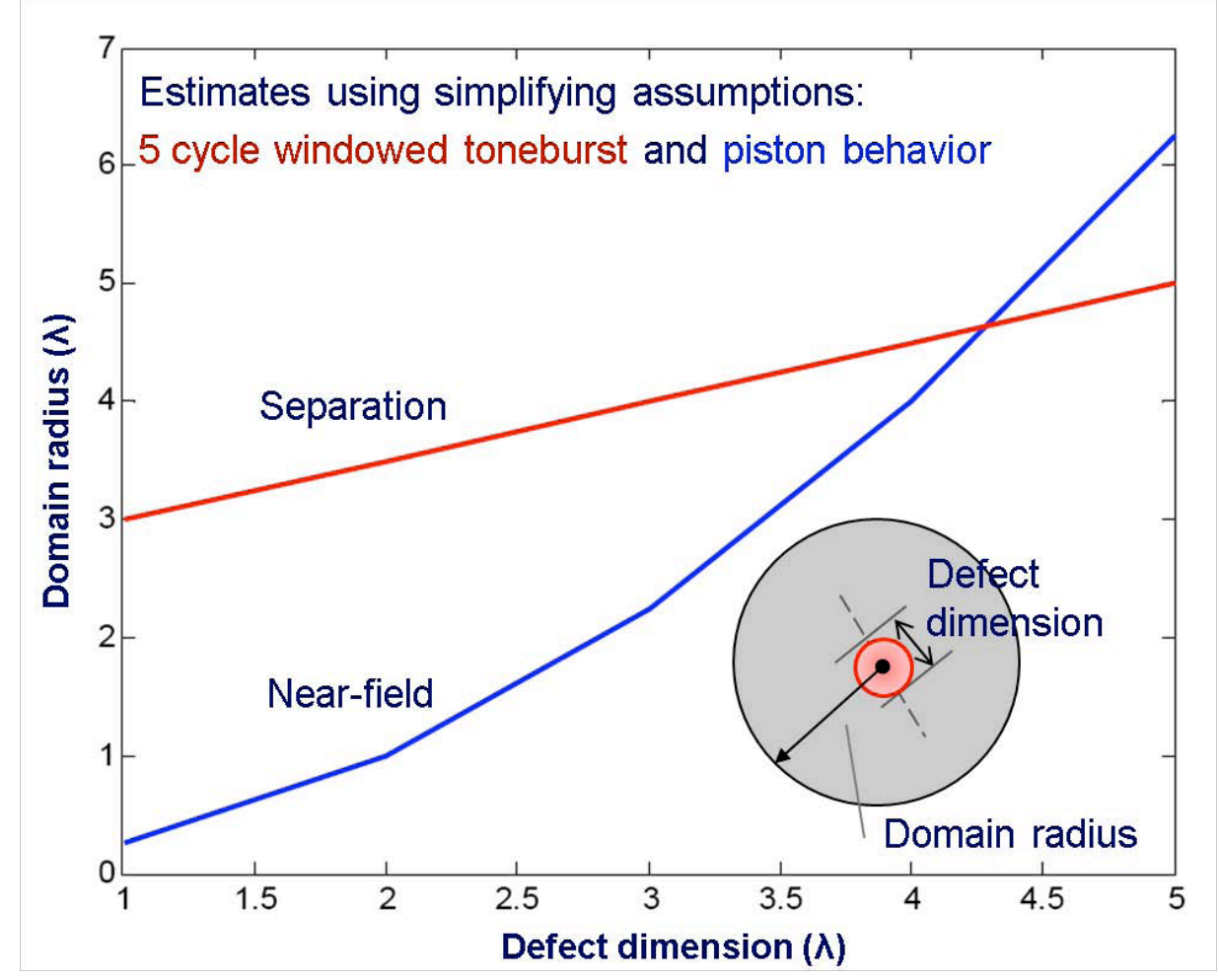

Fig. 6. Separation and near-field radius plotted versus the defect radius for the case where the excitation signal consists of a 5-cycle Hanning windowed toneburst. For a 2 lc long defect, the domain radius would need to be at least $3.5 \mathrm{lc}$ to ensure separation of incident and scattered signals, and at least $1 \mathrm{lc}$ to escape the near-field. Inset: an idealised circular domain with a circular defect. 

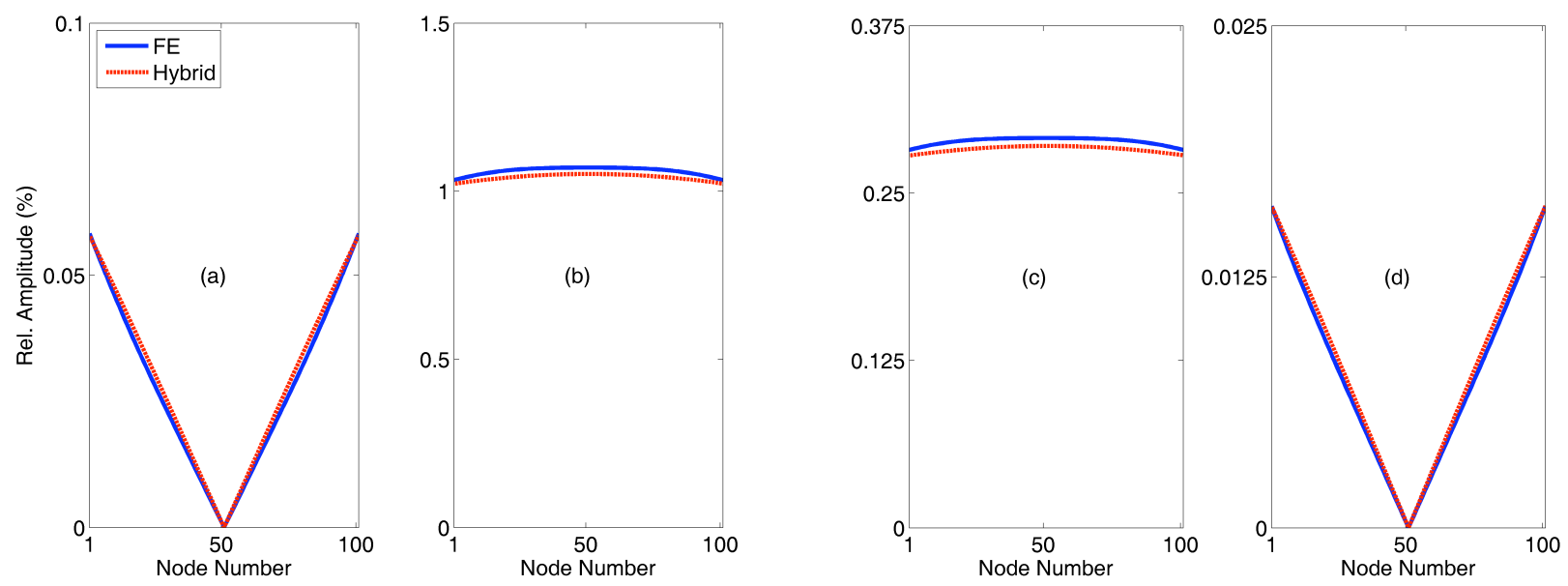

Fig. 7. Comparison of the hybrid interface model to full FE simulations for the back-scattering case. (a) $\mathrm{x}$ displacements and (b) y displacements for S-wave propagation; (c) $\mathrm{x}$ displacements and (d) y displacements for P-wave propagation. The comparison is performed along all the nodes on the right boundary of the source box, at the centre frequency of the signal in each case. 


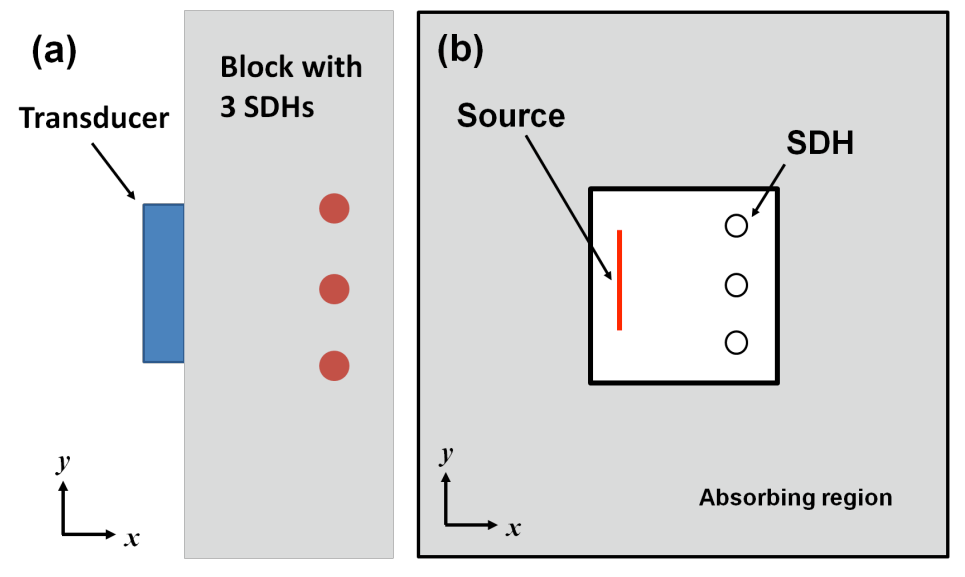

(c)

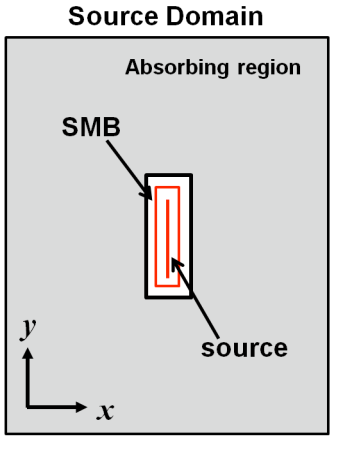

Defect Domain

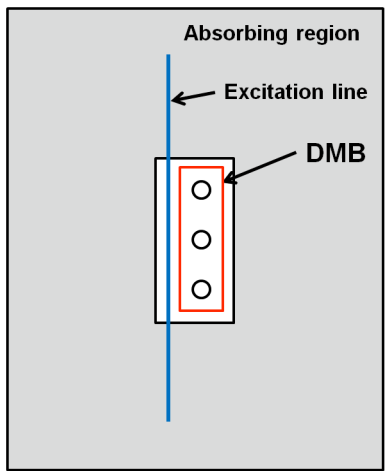

Fig. 8. Realistic example of prediction of scattering from a row of side drilled holes (SDHs). (a) illustration of test block with three side-drilled holes a transducer is placed on the left of the block and used to generate the test signal and receive the back-scattered signal. Illustration of the model of this case, showing (b) full FE domain and (c) the two boxes for the hybrid model. 


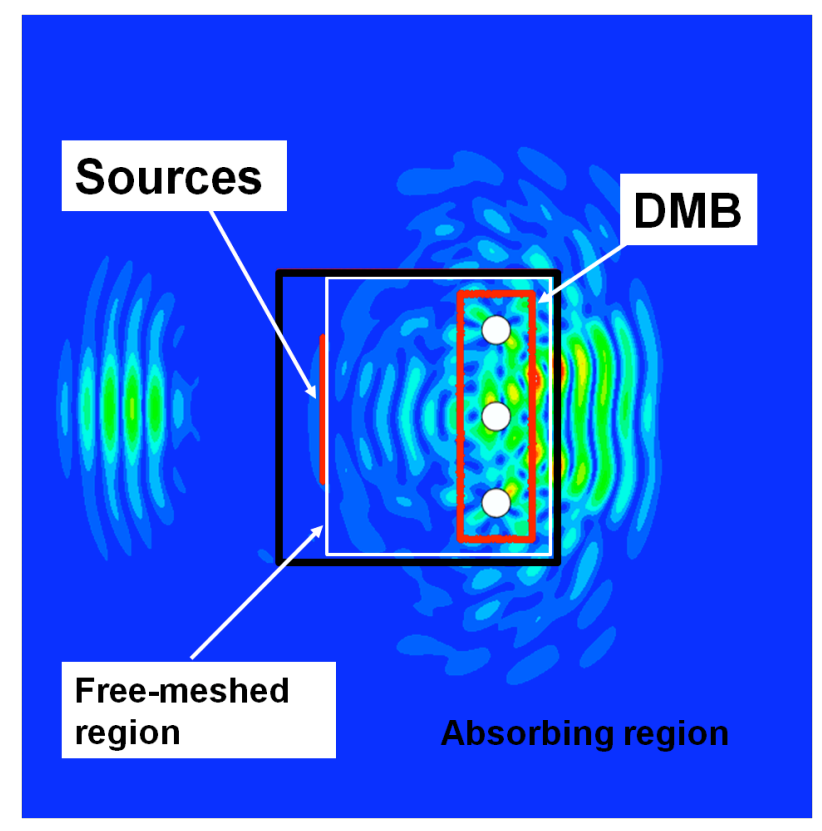

Fig. 9. Snapshot of the contour of total displacement magnitude, from FE simulation of the side-drilled holes case. Results shown are for the full FE calculation, showing the scattering field just after incidence of the wave pulse at the side drilled holes. 

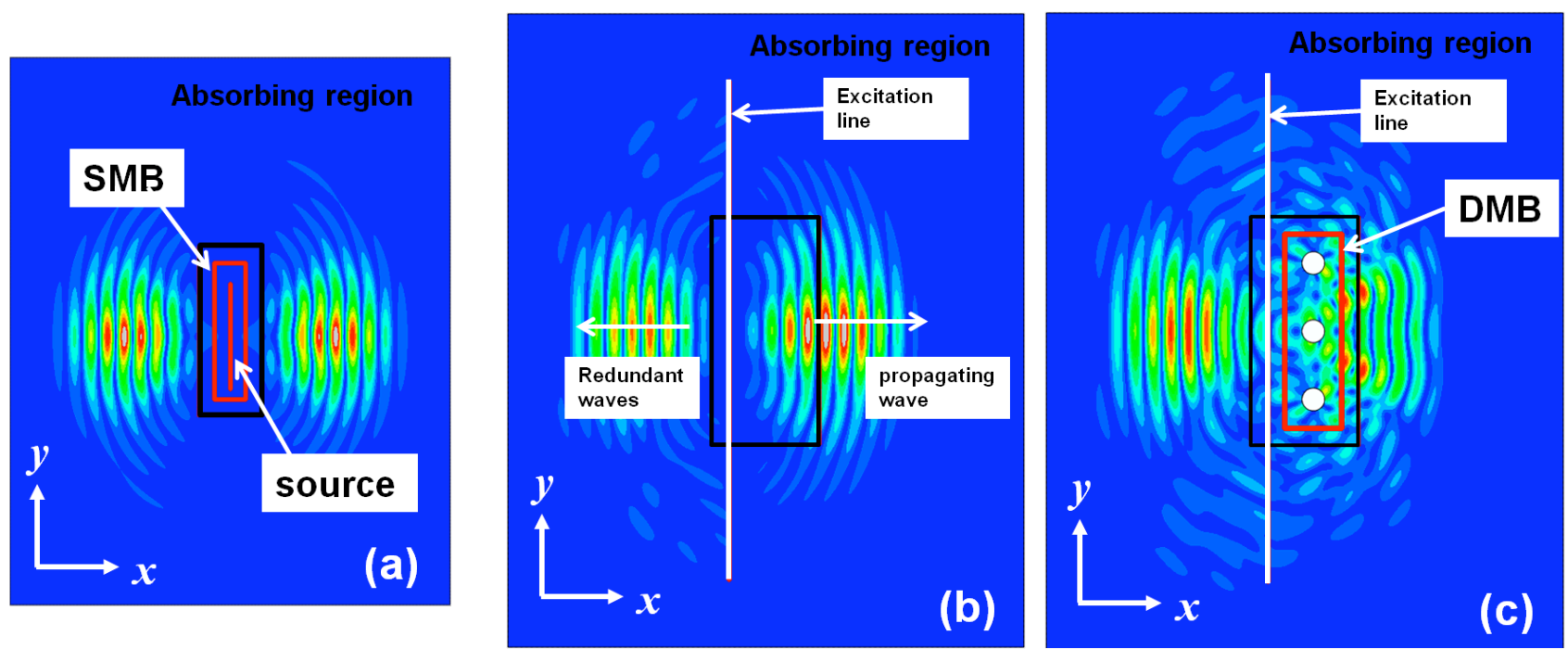

Figure 10. Snapshot of propagating wave through the two physically separated domains. (a) Source domain at $6 \mu \mathrm{sec}$ : propagating waves are monitored at the Source Monitoring Box (SMB) and used to calculate signals on secondary sources. (b) Defect domain without the SDHs at $8 \mu$ sec: propagationg waves are generated by imposing traction on the excitation line, representing the signal coming from the source box. For clarity, the field is shown for the case without the presence of SDHs. (c) Defect domain with the SDHs at $8 \mu \mathrm{sec}$ : The scattered waves are monitored at the DMB and fed into the hybrid interface to predict the signal at the receiver. 


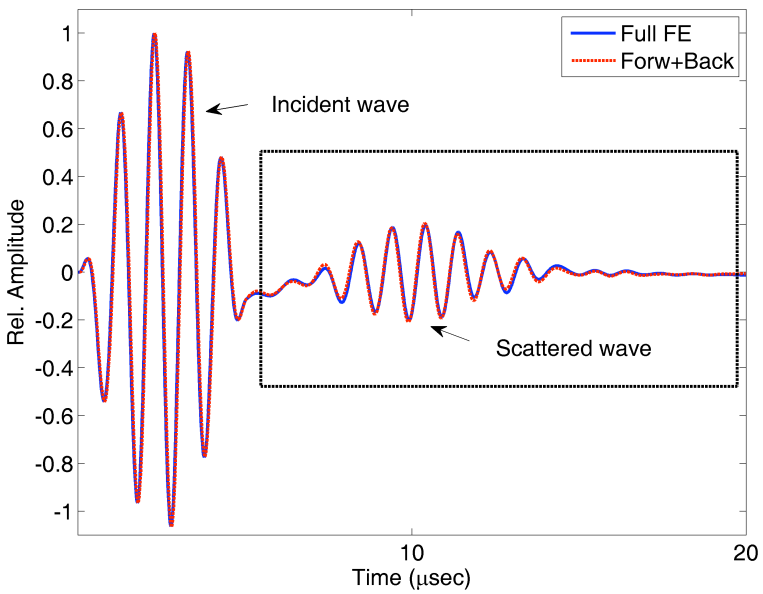

(a) Time signal

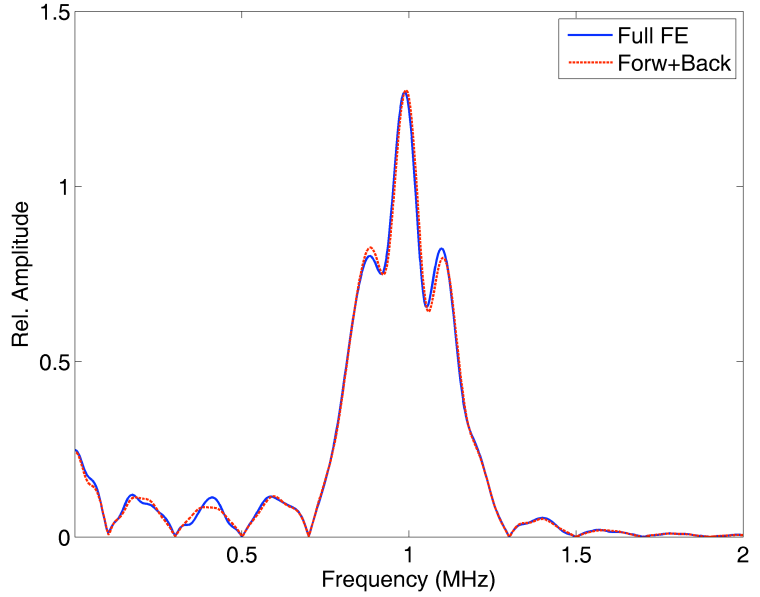

(b) Frequency signal

Fig. 11. Comparison of predicted amplitudes of the average y displacement at the nodes on the receiver, monitoring the waves scattering back from the side-drilled holes. Estimates of full FE (solid), back scattering only (dash-dot), and forward-backward (dashed) (a) time-domain; (b) frequency-domain. 


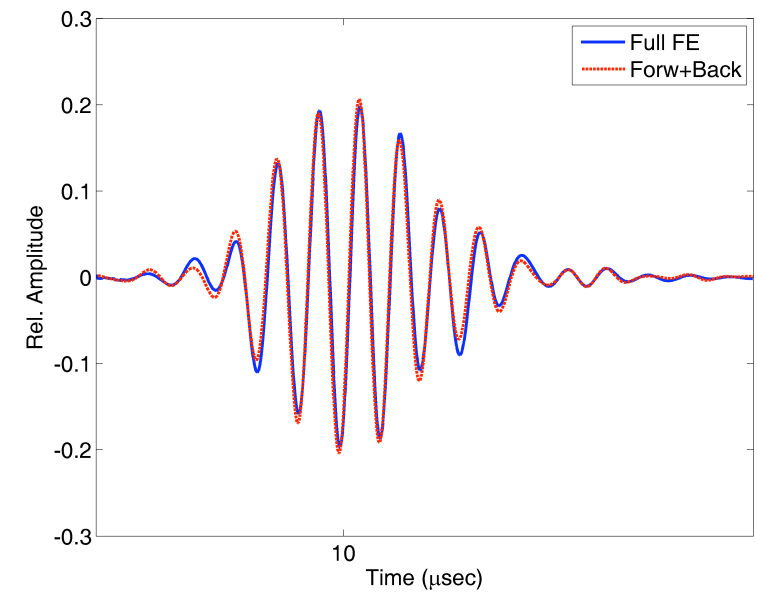

(a) Time signal

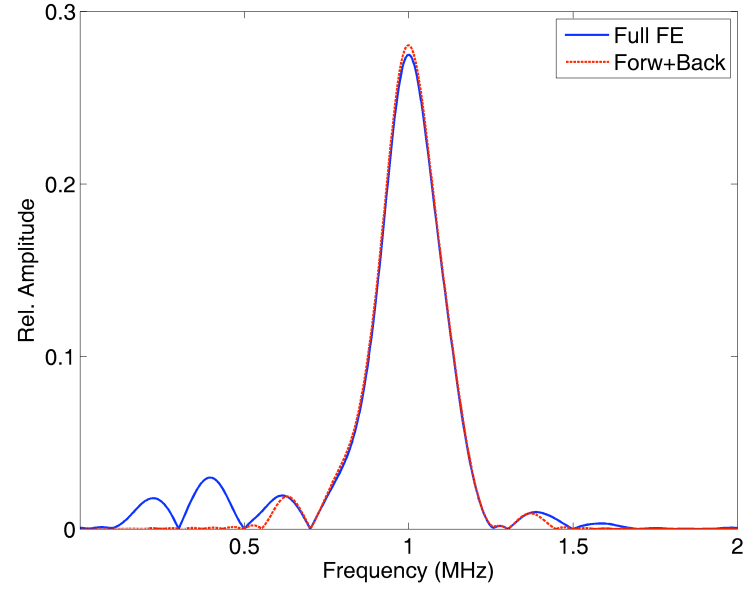

(b) Frequency signal

Fig. 12. Comparison as in Fig.11: Identical plots but only with the scattered wave, and the data from 5 to $20 \mu \mathrm{sec}$ inside the box in Fig.11a. 


\begin{tabular}{|l|l|l|l|l|}
\hline Error (\%) & a & b & c & d \\
\hline Fig. 5 & 4.03 & 2.32 & 0.49 & 6.93 \\
\hline Fig. 7 & 6.66 & 1.79 & 2.09 & 5.35 \\
\hline
\end{tabular}

Table 1. Errors averaged over 100 nodal points for each of the figure 5(a)-(d) and 7(a)-(b) 